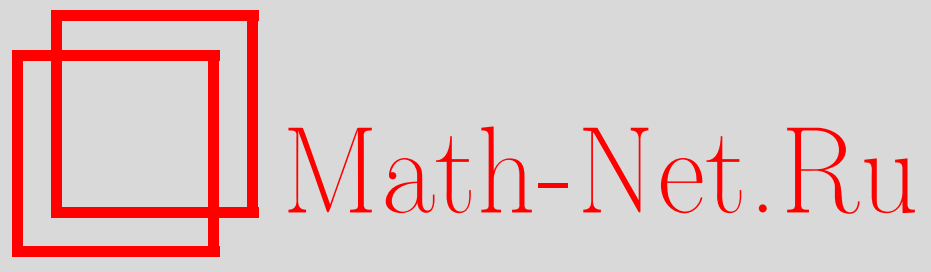

C. С. Гончаров, Об автоустойчивости относительно сильных конструктивизаций почти простых моделей, УМH, 2010, том 65, выпуск 5, 107-142

DOI: https://doi.org/10.4213/rm9373

Использование Общероссийского математического портала Math-Net.Ru подразумевает, что вы прочитали и согласны с пользовательским соглашением http://www . mathnet.ru/rus/agreement

Параметры загрузки:

IP : 3.85 .5 .30

26 апреля 2023 г., 16:49:27

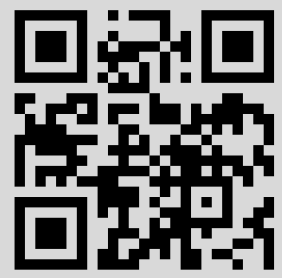


Посвящается столетнему юбилею основателя Сибирской школы алгебры и логики академика А. И. Мальцева

\title{
Об автоустойчивости относительно сильных конструктивизаций почти простых моделей
}

\author{
С. С. Гончаров
}

Вопросы автоустойчивости и алгоритмической размерности моделей восходят к работам А. И. Мальцева и А. Фрелиха-Дж. Шепердсона, в которых впервые был обнаружен эффект существования для моделей неэквивалентных, с точки зрения их алгоритмических свойств, вычислимых представлений. Исследованиям этих проблем в настоящее время посвящено большое число работ различных авторов. В данной статье исследуется вопрос о наследовании свойств автоустойчивости и неавтоустойчивости относительно сильных конструктивизаций на почти простых моделях при элементарных расширениях.

Библиография: 37 названий.

Ключевые слова: вычислимая модель, конструктивная модель, сильно конструктивная модель, автоустойчивость, простая модель, почти простая модель, эренфойхтова теория, разрешимая теория, разрешимая модель.

\section{СОДЕРЖАНИЕ}

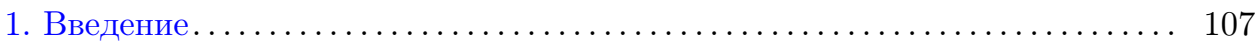

2. Предварительные сведения................................ 109

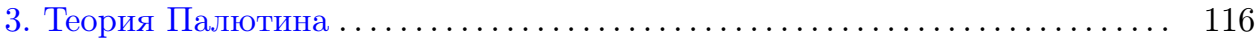

4. Эренфойхтова теория с автоустойчивой простой моделью........... 119

5. Теории с неавтоустойчивой простой моделью................... 122

Список литературы ........................................ 140

\section{1. Введение}

В работе А. И. Мальцева [1] было положено начало систематическому изучению конструктивных моделей. Для изучения вопроса о единственности конструктивной нумерации для моделей им было введено понятие рекурсивно

Работа выполнена при поддержке РФФИ (грант № 09-01-12140_офи_м), программы “Ведущие научные школы" (грант НШ-335.2008.1) и ФЦП "Научные и научно-педагогические кадры инновационной России" на 2009-2013 гг. (проект 02.740.11.0429).

(C) C. С. ГончАров, 2010 
устойчивой модели. Им было отмечено, что конечно порожденные алгебраические системы являются рекурсивно устойчивыми. В работе [2] было показано, что уже для бесконечномерного векторного пространства над полем рациональных чисел мы можем построить две различные конструктивизации такие, что в одной проблема линейной зависимости векторов разрешима, а в другой - неразрешима. В этой же работе А. И. Мальцев ввел понятия автоэквивалентных конструктивизаций и автоустойчивой модели. Им было показано, что бесконечномерное векторное пространство над полем рациональных чисел неавтоустойчиво. Впервые в других терминах проблема автоустойчивости для алгебраических замыканий полей была поставлена Ван-дер-Варденом. А. Фрелих и Дж. Шепердсон [3] ответили на вопрос Ван-дер-Вардена отрицательно, показав, что не всегда существует алгоритм, осуществляющий построение вычислимого изоморфизма между алгебраическими замыканиями поля, построенными на основе различных конструкций. С этих работ началось систематическое изучение проблемы описания автоустойчивых алгебраических систем. Вопрос о связи автоустойчивости с теоретико-модельными свойствами лежит в русле исследований этой проблематики, связанной с нахождением условий автоустойчивости моделей и сложности класса всех автоустойчивых моделей. Ю. Л. Ершовым [4] для построения теории конструктивных моделей было введено понятие сильно конструктивной модели; эквивалентное понятие разрешимой модели было введено М. Морли [5]. Эти понятия играют важную роль при изучении моделей разрешимых теорий. Для случая автоустойчивости относительно сильных конструктивизаций А. Т. Нуртазиным [6] был получен критерий автоустойчивости, который показывает тесную взаимосвязь проблемы автоустойчивости относительно сильных конструктивизаций с простотой модели. В настоящей работе исследуется вопрос существования полных разрешимых теорий с автоустойчивой простой моделью в конечном обогащении константами, но простая модель которых неавтоустойчива относительно сильных конструктивизаций, а также разрешимых полных теорий, у которых простая модель автоустойчива относительно сильных конструктивизаций, но существует неавтоустойчивая модель относительно сильных конструктивизаций этой теории, которая является простой моделью в конечном обогащении константами. Эти вопросы рассматриваются для различных классов теорий, в частности, малых теорий (т. е. со счетным числом простых моделей, со счетными насыщенными моделями), несчетно категоричных теорий и эренфойхтовых теорий. Эренфойхтовой называется полная теория, которая не является счетно категоричной и имеет конечное число типов изоморфизма счетных моделей. Класс эренфойхтовых теорий наименее изучен и понятен с точки зрения как их теоретико-модельных свойств, так и с точки зрения изучения свойств их вычислимых моделей таких, как вопросы о взаимосвязи вычислимости, разрешимости и автоустойчивости для класса их счетных моделей.

Изучению вопросов разрешимости и вычислимости счетных моделей эренфойхтовых и счетно категоричных теорий посвящены работы [5], [7]-[27] и др.

Обозначим число попарно неизоморфных счетных моделей теории $T$ через $I(\omega, T)$. Для класса эренфойхтовых теорий Р. Воотом [28] показано, что невозможен случай $I(\omega, T)=2$. Но для любого $n \geqslant 3$ существует эренфойхтова 
теория $T$ с $I(\omega, T)=n$. М. Г. Перетятькиным в [25] было показано, что разрешимая эренфойхтова теория имеет вычислимое семейство рекурсивных главных типов и ее простая модель разрешима. Из работы Е.А. Палютина [24] следует, что существует разрешимая счетно категоричная теория, у которой счетная модель разрешима, но не автоустойчива относительно сильных конструктивизаций. А отсюда и из конструкции А. Н. Гаврюшкина [12] легко получить пример эренфойхтовой теории с неавтоустойчивой относительно сильных конструктивизаций простой моделью на основе теоремы А. Т. Нуртазина из [6], где был найден критерий автоустойчивости моделей относительно сильных конструктивизаций. Направление, связанное с изучением спектра вычислимых моделей в классе счетных моделей, представлено в работах А.Н. Гаврюшкина [11]-[16]. Тесно связаны с этой проблемой описания тьюринговых степеней, в которых модели эренфойхтовой теории имеют вычислимые (разрешимые) относительно этих степеней представления. Менее изучены вопросы об автоустойчивости вычислимых моделей эренфойхтовых теорий. Интересен вопрос о связи автоустойчивости и автоустойчивости относительно сильных конструктивизаций для различных счетных моделей полных разрешимых элементарных теорий. Заметим, что все почти простые разрешимые модели будут 0 -автоустойчивыми, но не все из них автоустойчивы. В то же время произвольные разрешимые модели могут быть не $\Delta_{1}^{1}$-автоустойчивы относительно сильных конструктивизаций.

Стандартные определения и обозначения взяты из [7], [29]-[31].

\section{2. Предварительные сведения}

Прежде чем переходить к основным результатам, напомним базовые понятия логики и теории моделей, а на их основе определим понятия теории вычислимых моделей.

Как и в естественном языке, для определения формального языка логики первого порядка требуется определить алфавит этого языка. Ядром языка логики первого порядка является сигнатура. Напомним определение понятия сигнатуры. Сигнатура языка определяет набор имен для основных отношений, операций и выделенных элементов, а также местность (арность) соответствующих отношений и операций.

Сигнатурой называется набор $\Sigma=\left\langle\Sigma_{R}, \Sigma_{F}, \Sigma_{C}, \rho\right\rangle$, состоящий из множеств

$\Sigma_{R}$ - символов (имен) для основных отношений (предикатов),

$\Sigma_{F}-$ символов (имен) для основных операций (функций),

$\Sigma_{C}$ - символов (имен) для основных констант (выделенных элементов), и функции $\rho$, сопоставляющей именам предикатов и операций их арность, т. е. местность соответствующих операций и отношений, $\rho: \Sigma_{R} \cup \Sigma_{F} \rightarrow \mathbb{N}$.

Часто мы будем записывать сигнатуру в виде

$$
\left\langle P_{1}, \ldots, P_{n} ; G_{1}, \ldots, G_{s} ; c_{0}, \ldots, c_{r} ; \rho\right\rangle
$$

или

$$
\left\langle P_{1}^{m_{1}}, \ldots, P_{n}^{m_{k}} ; G_{1}^{n_{1}}, \ldots, G_{s}^{n_{s}} ; c_{0}, \ldots, c_{z}\right\rangle
$$


Моделью $\mathfrak{A}$ сигнатурь $\Sigma$ называем пару $\left\langle A ; \operatorname{int}_{\Sigma}^{A}\right\rangle$, состоящую из непустого множества $A$ и $\operatorname{int}_{\Sigma}^{A}$ - отображения из множества имен сигнатуры этой модели в множество отношений и операций на $A$ и элементов из $A$, которую мы будем называть интерпретацией для имен из сигнатуры $\Sigma$. Интерпретация int ${ }_{\Sigma}^{A}$ представляется функцией, определенной на множестве $\Sigma_{R} \cup \Sigma_{F} \cup \Sigma_{C}$, и такой, что

$$
\operatorname{int}_{\Sigma}^{A}: \Sigma_{R} \rightarrow \mathscr{P}\left(A^{\mathbb{N}}\right)
$$

где $\mathscr{P}\left(A^{n}\right)$ - множество всех подмножеств $A^{n}$, а $\mathscr{P}\left(A^{\mathbb{N}}\right)=\bigcup_{n} \mathscr{P}\left(A^{n}\right)$,

$$
\operatorname{int}_{\Sigma}^{A}: \Sigma_{F} \rightarrow \bigcup_{n} F\left(A^{n}, A\right)
$$

где $F\left(A^{n}, A\right)$ - множество всех функций из $A^{n}$ в $A$, и

$$
\operatorname{int}_{\Sigma}^{A}: \Sigma_{C} \rightarrow A
$$

причем выполнены следующие условия относительно местности определяемых отношений и операций:

$$
\operatorname{int}_{\Sigma}^{A}(P) \subseteq A^{n}, \quad \text { если } \rho(P)=n \text { и } P \in \Sigma_{R},
$$

и

$$
\operatorname{int}_{\Sigma}^{A}(G): A^{m} \rightarrow A, \quad \text { если } \rho(G)=m \text { и } G \in \Sigma_{F} .
$$

Множество $A$ в этом случае называется основным множеством модели $\mathfrak{A}$ и, как правило, обозначается через $|\mathfrak{A}|$. Часто вместо термина "алгебраическая система" используют термины "модель" или "структура".

Будем записывать модели конечной сигнатуры в виде

$$
\mathfrak{A}=\left\langle A,\left(P_{1}^{m_{1}}\right)_{\mathfrak{A}}, \ldots,\left(P_{k}^{m_{k}}\right)_{\mathfrak{A}} ;\left(G_{1}^{n_{1}}\right)_{\mathfrak{A}}, \ldots,\left(G_{s}^{n_{s}}\right)_{\mathfrak{A}} ;\left(c_{0}\right)_{\mathfrak{A}}, \ldots,(c)_{\mathfrak{A}}\right\rangle,
$$

указывая непосредственно через индекс модель, в которой они интерпретируются. Если из контекста ясно, в какой модели интерпретируются эти сигнатурные символы, а также определена их местность, то будем опускать эти индексы в задании модели.

Пусть заданы две модели

$$
\mathfrak{A}=\left\langle A,\left(P_{1}^{m_{1}}\right)_{\mathfrak{A}}, \ldots,\left(P_{k}^{m_{k}}\right)_{\mathfrak{A}} ;\left(G_{1}^{n_{1}}\right)_{\mathfrak{A}}, \ldots,\left(G_{s}^{n_{s}}\right)_{\mathfrak{A}} ;\left(c_{0}\right)_{\mathfrak{A}}, \ldots,\left(c_{r}\right)_{\mathfrak{A}}\right\rangle
$$

И

$$
\mathfrak{B}=\left\langle B,\left(P_{1}^{m_{1}}\right)_{\mathfrak{B}}, \ldots,\left(P_{k}^{m_{k}}\right)_{\mathfrak{B}} ;\left(F_{1}^{n_{1}}\right)_{\mathfrak{B}}, \ldots,\left(F_{s}^{n_{s}}\right)_{\mathfrak{B}} ;\left(c_{0}\right)_{\mathfrak{B}}, \ldots,\left(c_{r}\right)_{\mathfrak{B}}\right\rangle
$$

одной сигнатуры.

Определим теперь важное для теории моделей и алгебры понятие изоморфизма алгебраических систем одной и той же сигнатуры.

Изоморфизмом $\varphi: \mathfrak{A} \rightarrow \mathfrak{B}$ модели

$$
\mathfrak{A}=\left\langle A,\left(P_{1}^{m_{1}}\right)_{\mathfrak{A}}, \ldots,\left(P_{k}^{m_{k}}\right)_{\mathfrak{A}} ;\left(F_{1}^{n_{1}}\right)_{\mathfrak{A}}, \ldots,\left(F_{s}^{n_{s}}\right)_{\mathfrak{A}} ;\left(c_{0}\right)_{\mathfrak{A}}, \ldots,\left(c_{r}\right)_{\mathfrak{A}}\right\rangle
$$


в модель

$$
\mathfrak{B}=\left\langle B,\left(P_{1}^{m_{1}}\right)_{\mathfrak{B}}, \ldots,\left(P_{k}^{m_{k}}\right)_{\mathfrak{B}} ;\left(F_{1}^{n_{1}}\right)_{\mathfrak{B}}, \ldots,\left(F_{s}^{n_{s}}\right)_{\mathfrak{B}} ;\left(c_{0}\right)_{\mathfrak{B}}, \ldots,\left(c_{r}\right)_{\mathfrak{B}}\right\rangle
$$

той же сигнатуры назовем разнозначное отображение $\varphi: A \rightarrow \mathfrak{B}$ такое, что:

1) для любого сигнатурного константного символа $c_{i}, i \leqslant r$, выполнено равенство $\varphi\left(\left(c_{i}\right)_{\mathfrak{A}}\right)=\varphi\left(\left(c_{i}\right)_{\mathfrak{B}}\right)$;

2) для любого сигнатурного предикатного символа $P_{i}, i \leqslant k$, местности $m_{i}$ и любых элементов $\left\langle a_{1}, \ldots, a_{m_{i}}\right\rangle \in A^{m_{i}}$ выполнено $\left\langle a_{1}, \ldots, a_{n_{i}}\right\rangle \in\left(P_{i}\right)_{\mathfrak{A}}$, если и только если $\left\langle\varphi\left(a_{1}\right), \ldots, \varphi\left(a_{n}\right)\right\rangle \in\left(P_{i}\right)_{\mathfrak{B}}$;

$3)$ для любого сигнатурного функционального символа $F_{i}, i \leqslant s$, местности $n_{i}$ и любых элементов $\left\langle a_{1}, \ldots, a_{n}\right\rangle \in A^{n_{i}}$ выполнено равенство $F_{i \mathfrak{B}}\left(\varphi\left(a_{1}\right)\right.$, $\left.\ldots, \varphi\left(a_{n_{i}}\right)\right)=\varphi\left(F_{i} \mathfrak{A}\left(a_{1}, \ldots, a_{n_{i}}\right)\right)$.

Если, кроме того, отображение $\varphi: A \rightarrow B$ является отображением на все множество, $\varphi: A \rightarrow \mathfrak{B}$, то такие модели называем изоморфными. Легко видеть, что при этом обратное отображение также является изоморфизмом. В этом случае называем модели

$$
\mathfrak{A}=\left\langle A,\left(P_{1}^{m_{1}}\right)_{\mathfrak{A}}, \ldots,\left(P_{k}^{m_{k}}\right)_{\mathfrak{A}} ;\left(F_{1}^{n_{1}}\right)_{\mathfrak{A}}, \ldots,\left(F_{s}^{n_{s}}\right)_{\mathfrak{A}} ;\left(c_{0}\right)_{\mathfrak{A}}, \ldots,\left(c_{r}\right)_{\mathfrak{A}}\right\rangle
$$

и

$$
\mathfrak{B}=\left\langle B,\left(P_{1}^{m_{1}}\right)_{\mathfrak{B}}, \ldots,\left(P_{k}^{m_{k}}\right)_{\mathfrak{B}} ;\left(F_{1}^{n_{1}}\right)_{\mathfrak{B}}, \ldots,\left(F_{s}^{n_{s}}\right)_{\mathfrak{B}} ;\left(c_{0}\right)_{\mathfrak{B}}, \ldots,\left(c_{r}\right)_{\mathfrak{B}}\right\rangle
$$

изоморфными.

В алгебре и теории моделей обычно изучаются свойства алгебраических систем с точностью до изоморфизма и мы не будем различать изоморфные модели. Как хорошо известно, изоморфные модели не различимы на языке логики первого порядка [31].

Определим синтаксис нашего точного математического языка. Для этого вводятся понятия термов и формул языка логики первого порядка [7]. Фиксируем алфавит, состоящий из символов нашего языка. Конечные последовательности символов из этого алфавита являются словами, но, как и в обычном языке, не все такие последовательности осмысленны. Нас интересуют лишь те слова, которые являются термами и формулами фиксированной сигнатуры, так как они позволяют описывать на точном математическом языке с корректной интерпретацией свойства моделей и формулировать теоремы и аксиомы теорий.

Теперь все готово для определения по вычислимой сигнатуре гёделевской нумерации термов и формул, что позволит средствами классической вычислимости исследовать алгоритмические свойства моделей и их элементарных теорий.

Зафиксируем множество переменных $\mathscr{V}$ нашего языка. Без ограничения общности будем использовать в качестве множества переменных $\mathscr{V}$ множество $\left\{v_{0}, v_{1}, \ldots, v_{n}, \ldots\right\}$.

Для применения классической теории вычислимости к вопросам разрешимости алгоритмических свойств на модели построим гёделевскую нумерацию термов и формул фиксированной сигнатуры. Вначале рассмотрим некоторые 
нумерации всех предикатных, функциональных и константных символов этой сигнатуры. Пусть сигнатура задана в виде

$$
\sigma=\left\langle P_{0}^{n_{0}}, \ldots, P_{k}^{n_{k}}, \ldots ; F_{0}^{m_{0}}, \ldots, F_{s}^{m_{s}}, \ldots ; c_{0}, \ldots, c_{n}, \ldots\right\rangle .
$$

Потребуем, чтобы для нее выполнялось свойство вычислимости арности этих символов, а именно, чтобы существовали вычислимые функции $[n]$ и $[m]$ такие, что $[n](i)=n_{i}$, где $n_{i}$ - арность предикатного символа $P_{i}$, а $[m](i)=m_{i}$, где $m_{i}$ - арность функционального символа $F_{i}$. В случае, если функции $[n]$ и $[m]$ вычислимы, будем говорить, что наш язык вычислим. Заметим, что если сигнатура конечна, то наш язык всегда будет вычислим.

Рассмотрим также обогащение $\sigma_{\mathbb{N}}$ нашей сигнатуры $\sigma$ новыми константными символами:

$$
\sigma_{\mathbb{N}} \leftrightharpoons \sigma \cup\left\langle a_{0}, a_{1}, \ldots\right\rangle
$$

Пусть $L$ и $L_{\mathbb{N}}$ являются языками первого порядка соответственно сигнатуры $\sigma$ и $\sigma_{\mathbb{N}}$, включающими все формулы и термы соответствующих сигнатур с переменными из множества $\mathscr{V}$. Полагаем, что $P_{0}$ будет использоваться в наших языках $L$ и $L_{\mathbb{N}}$ в качестве символа равенства.

Построим гёделевскую нумерацию всех термов и формул языка первого порядка с переменными из $\mathscr{V}$ так, что по номеру терма или формулы можно вычислить всю информацию о строении соответствующего объекта в нашем языке, следуя в нашем определении монографии [7].

Определим в качестве гёделевской нумерации функцию $\gamma$, отображающую множество $\operatorname{Term}_{\sigma_{\mathbb{N}}}(\mathscr{V}) \cup$ Form $_{\sigma_{\mathbb{N}}}(\mathscr{V})$, являющееся объединением множества термов $\operatorname{Term}_{\sigma_{\mathbb{N}}}(\mathscr{V})$ и множества формул $\operatorname{Form}_{\sigma_{\mathbb{N}}}(\mathscr{V})$ сигнатуры $\sigma_{\mathbb{N}}$ с переменными из множества $\mathscr{V}$, разнозначно в множество натуральных чисел $\mathbb{N}$. Построим данную функцию индукцией по построению соответственно термов и формул нашего языка.

Пусть примитивно рекурсивные функции $c, l, r$ из [32] задают нумерацию упорядоченных пар натуральных чисел, т. е. $c: \mathbb{N}^{2} \rightarrow \mathbb{N}$ и для любых $n, m$ выполнены равенства $c(l(n), r(n))=n, l c(n, m)=n$ и $r c(n, m)=m$.

Вначале определим номера для термов сигнатуры $\sigma_{\mathbb{N}}$ с переменными из $\mathscr{V}-$ функцию на $\operatorname{Term}_{\sigma_{\mathbb{N}}}(\mathscr{V})$ :

1) $\gamma\left(v_{i}\right)=c(0, c(0, i))$;

2) $\gamma\left(c_{i}\right)=c(0, c(1, i))$ для $i$ таких, что $c_{i} \in \sigma$;

$\left.2^{\prime}\right) \gamma\left(a_{i}\right)=c(0, c(2, i))$ для $i$ таких, что $a_{i} \in \sigma_{\mathbb{N}}$;

$3)$ если терм $t$ имеет вид $F_{i}\left(t_{1}, \ldots, t_{m_{i}}\right)$, где $F_{i}$ является $m_{i}$-арным предикатным символом нашей сигнатуры, а термы $t_{1}, \ldots, t_{m_{i}}$ уже имеют гёделевские номера $\gamma\left(t_{1}\right)=l_{1}, \ldots, \gamma\left(t_{m_{i}}\right)=l_{m_{i}}$, то номер нашего терма получается следующим образом:

$$
\gamma(t)=c\left(0, c\left(i+3, c^{m_{i}}\left(l_{1}, \ldots, l_{m_{i}}\right)\right)\right) .
$$

Нетрудно видеть, что множество номеров термов рекурсивно и даже примитивно рекурсивно, и кроме того, по номеру можно распознать, какие переменные и константы входят в этот терм, а также найти номера всех подтермов.

Доопределим теперь гёделевскую нумерацию на множестве формул сигнатуры $\sigma_{\mathbb{N}}$. 
Определим функцию $\gamma$ также индукцией по построению формул:

1) если $t$ и $q$ - термы и мы знаем их номера $\gamma(t)=n$ и $\gamma(q)=m$, то определим номер $\gamma(t=q) \leftrightharpoons c(1, c(0, c(n, m)))$;

2) если $P_{i}$ является $n_{i}$-арным предикатным символом нашей сигнатуры, a $t_{1}, \ldots, t_{n_{i}}$ - термы с гёделевскими номерами $\gamma\left(t_{1}\right)=l_{1}, \ldots, \gamma\left(t_{n_{i}}\right)=l_{n_{i}}$, то полагаем $\gamma\left(P_{i}\left(t_{1}, \ldots, t_{n_{i}}\right)\right) \leftrightharpoons c\left(1, c\left(1, c\left(i+1, c^{n_{i}}\left(l_{1}, \ldots, l_{n_{i}}\right)\right)\right)\right)$ и $\gamma\left(t_{1}=t_{2}\right)=$ $c\left(1, c\left(1, c\left(0, c\left(l_{1}, l_{2}\right)\right)\right)\right)$;

$3)$ если $\varphi$ и $\psi$ - формулы с гёделевскими номерами $\gamma(\varphi)=n$ и $\gamma(\psi)=m$, то полагаем

$$
\begin{aligned}
\gamma((\varphi \& \psi)) & \leftrightharpoons c(1, c(2, c(n, m))) \\
\gamma((\varphi \vee \psi)) & \leftrightharpoons c(1, c(3, c(n, m))), \\
\gamma((\varphi \rightarrow \psi)) & \leftrightharpoons c(1, c(4, c(n, m))), \\
\gamma(\neg \varphi) & \leftrightharpoons c(1, c(5, n)), \\
\gamma\left(\left(\exists v_{i}\right) \varphi\right) & \leftrightharpoons c(1, c(6, c(i, n))), \\
\gamma\left(\left(\forall v_{i}\right) \varphi\right) & \leftrightharpoons c(1, c(7, c(i, n))) .
\end{aligned}
$$

Индукцией по сложности термов и формул можно легко показать, что каждый терм и каждая формула получат свои номера и эта функция разнозначна. Кроме того, можно вычислить всю информацию о строении формул, их подформул, вхождениях переменных и констант по их номерам.

С каждым подмножеством $S \subseteq L_{\mathbb{N}}$ языка $L_{\mathbb{N}}$ мы связываем множество гёделевских номеров $\gamma(S)$ всех элементов из $S$. Множество $S$ называется разрешимым, если множество гёделевских номеров его элементов рекурсивно, и множество $S$ вычислимо перечислимо, если множество гёделевских номеров его элементов рекурсивно перечислимо.

Введем следующие обозначения. Пусть $\mathfrak{M}$ - модель сигнатуры $\sigma$. Обозначим через $\operatorname{Th}(\mathfrak{M})$ элементарную теорию этой модели. Если $\nu$ - нумерация основного множества модели $\mathfrak{M}$, то назовем пару $(\mathfrak{M}, \nu)$ нумерованной моделью. Если $(\mathfrak{M}, \nu)$ - нумерованная модель, то рассмотрим обогащение $\mathfrak{M}_{\nu}$ модели $\mathfrak{M}$ до сигнатуры $\sigma_{\mathbb{N}}$, взяв в качестве значения константы $a_{i}$ элемент $\nu(i)$ для любого $i \in \mathbb{N}$.

Обозначим через $\operatorname{Th}(\mathfrak{M}, \nu)$ элементарную теорию модели $\mathfrak{M}_{\nu}$, т. е. множество предложений сигнатуры $\sigma_{\mathbb{N}}$, истинных в модели $\mathfrak{M}_{\nu}$.

Нумерованная модель $(\mathfrak{M}, \nu)$ называется сильно конструктивной [4], если элементарная теория $\operatorname{Th}(\mathfrak{M}, \nu)$ сигнатуры $\sigma_{\mathbb{N}}$ разрешима.

Пусть

$$
\begin{gathered}
D(\mathfrak{M}, \nu)=\left\{\varphi \mid \varphi \text { является бескванторным предложением сигнатуры } \sigma_{\mathbb{N}}\right. \\
\text { и выполнено условие } \mathfrak{M} \models \varphi\} .
\end{gathered}
$$

Нумерованная модель $(\mathfrak{M}, \nu)$ называется конструктивной, если рекурсивно множество

$$
\begin{gathered}
\bar{D}(\mathfrak{M}, \nu) \leftrightharpoons\left\{\varphi \left(c_{m_{1}}, \ldots, c_{m_{k}} \mid \varphi\left(x_{1}, \ldots, x_{m_{k}}\right)-\right.\right.\text { атомная формула } \\
\text { и выполнено условие } \left.\mathfrak{M} \models \varphi\left(\nu m_{1}, \ldots, \nu m_{k}\right)\right\} .
\end{gathered}
$$


Здесь формула называется атомной, если в нее входит не более одного предикатного или функционального символа, а также не входят логические связки и кванторы.

Заметим, что нумерованная модель $(\mathfrak{M}, \nu)$ является конструктивной, если и только если множество $D(\mathfrak{M}, \nu)$ разрешимо, т. е. существует алгоритм проверки истинности для бескванторных формул на элементах этой модели. Ясно также, что любая сильно конструктивная модель является конструктивной, но обратное не верно. Заметим, кроме того, что элементарная теория сильно конструктивной модели будет разрешимой.

Мы будем говорить, что модель (сильно) конструктивизируема, если она имеет (сильную) конструктивизацию.

Введем также эквивалентные понятия разрешимой и вычислимой (рекурсивной) модели.

Пусть $\mathfrak{A}$ - модель сигнатуры $\sigma$, а ее основное множество $A$ является подмножеством множества натуральных чисел $\mathbb{N}$. Рассмотрим в этом случае сигнатуру $\sigma_{A}$, полученную из сигнатуры $\sigma$ добавлением константных символов $\left\{a_{i} \mid i \in A\right\}$. Заметим, что сигнатура $\sigma_{A}$ является частью сигнатуры $\sigma_{\mathbb{N}}$. Рассмотрим обогащение $\mathfrak{A}_{A}$ модели $\mathfrak{A}$ до сигнатуры $\sigma_{A}$, взяв в качестве значения константы $a_{i}$ элемент $i$ из $A$ для каждого $i \in A$.

Обозначим через $\operatorname{Th}\left(\mathfrak{A}_{A}\right)$ элементарную теорию обогащенной константами модели $\mathfrak{A}_{A}$, т. е. множество предложений сигнатуры $\sigma_{A}$, истинных в модели $\mathfrak{A}_{A}$.

Модель $\mathfrak{A}$ называется разрешимой [5], если элементарная теория $\operatorname{Th}\left(\mathfrak{A}_{A}\right)$ модели $\mathfrak{A}_{A}$ сигнатуры $\sigma_{A}$ разрешима.

Иногда модель, для которой существует изоморфная ей разрешимая модель, также будет называться разрешимой.

Пусть $D\left(\mathfrak{A}_{A}\right)=\left\{\varphi \mid \varphi\right.$ является бескванторным предложением сигнатуры $\sigma_{A}$ и выполнено условие $\mathfrak{M} \models \varphi\}$.

Модель $\mathfrak{A}$ называется вычислимой (рекурсивной) [2], если множество $A$ рекурсивно и множество бескванторных предложений $D\left(\mathfrak{A}_{A}\right)$ является разрешимым.

Легко заметить, что, выбрав для каждого элемента в конструктивной модели наименьший номер и индуцировав на это множество структуру нашей модели, получим вычислимую модель, причем если первоначальная модель была сильно конструктивной, то и полученная модель будет сильно конструктивной. Обратно, по вычислимой модели легко построить ее конструктивизацию, взяв в качестве нумерации любое рекурсивное перечисление ее основного множества $A$, причем она будет сильной, если наша модель разрешима.

Пусть $(\mathfrak{M}, \nu)$ и $(\mathfrak{M}, \mu)$ - две нумерованные модели для модели $\mathfrak{M}$. Будем говорить, что нумерации $\nu$ и $\mu$ модели $\mathfrak{M}$ рекурсивно эквивалентны, если существуют рекурсивные функции $f$ и $g$ такие, что $\nu=\mu f$ и $\mu=\nu g$. Для конструктивизаций $\nu$ и $\mu$ достаточно требовать существования рекурсивной функции $f$ такой, что $\nu=\mu f$. А.И. Мальцев [2] ввел более слабое понятие автоэквивалентности. Будем говорить, что конструктивизации $\nu$ и $\mu$ модели $\mathfrak{M}$ автоэквивалентны, если существуют рекурсивная функция $f$ и автоморфизм $\lambda$ модели $\mathfrak{M}$ такие, что $\lambda \nu=\mu f$. Модель называется автоустойчивой (относительно сильных конструктивизаций), если для любых двух (сильных) 
конструктивизаций $\nu_{1}$ и $\nu_{2}$ модели $\mathfrak{M}$ существуют автоморфизм $\alpha$ модели $\mathfrak{M}$ и общерекурсивная функция $f$ такие, что $\alpha \nu_{1}=\nu_{2} f$.

Напомним, что теория называется счетно категоричной, если она полна и имеет единственную с точностью до изоморфизма счетную модель, а эренфойхтовой называется теория, если она полна и имеет конечное число счетных моделей, но не является счетно категоричной.

Модель, полученная из модели $\mathfrak{M}$ добавлением в сигнатуру констант для конечного набора $A$ элементов из $\mathfrak{M}$, будем называть конечным обогащением модели $\mathfrak{M}$ константами и обозначать $(\mathfrak{M}, \bar{a})_{a \in A}$, где $\bar{a}-$ конечный набор элементов $\mathfrak{M}$.

Назовем модель $\mathfrak{M}$ простой моделью полной теории $T$, если она элементарно вкладывается в любую другую модель теории $T$. Модель $\mathfrak{M}$ атомная, если для любого набора элементов $a_{1}, \ldots, a_{n} \in|\mathfrak{M}|$ существует формула $\psi\left(x_{1}, \ldots, x_{n}\right)$ такая, что $\mathfrak{M} \models \psi\left(a_{1}, \ldots, a_{n}\right)$ и для любой формулы $\phi\left(x_{1}, \ldots, x_{n}\right)$, если $\mathfrak{M} \models$ $\phi\left(a_{1}, \ldots, a_{n}\right)$, то

$$
\mathfrak{M} \models\left(\forall x_{1}\right) \ldots\left(\forall x_{n}\right)\left(\psi\left(x_{1}, \ldots, x_{n}\right) \rightarrow \phi\left(x_{1}, \ldots, x_{n}\right)\right),
$$

а такая формула $\psi\left(x_{1}, \ldots, x_{n}\right)$ называется полной формулой теории этой модели.

По критерию Воота (см. [31]) счетная модель является простой, если и только если она атомная.

Будем называть модель почти простой, если она будет простой в некотором конечном обогащении константами.

Вопросы существования конструктивизаций для моделей и их автоустойчивости и алгоритмических размерностей лежат в центре основных исследований по теории вычислимых моделей [7], [17]. Ряд вопросов о существовании сильных конструктивизаций для моделей элементарных теорий и простых моделей, а также проблема автоустойчивости относительно сильных конструктивизаций были решены в следующих теоремах, которые будут использоваться в настоящей статье.

Начнем с важной теоремы А. Т. Нуртазина, которая показывает, что автоустойчивыми относительно сильных конструктивизаций могут быть лишь почти простые модели, и дает для них критерий автоустойчивости относительно сильных конструктивизаций.

Теорема 1 (критерий А. Т. Нуртазина [6]). Пусть $\mathfrak{M}$ - сильно конструктивная модель полной теории Т. Тогда следующие условия эквивалентны:

1) $\mathfrak{M}$ автоустойчива относительно сильных конструктивизаций;

2) существует конечная последовательность $\bar{a}$ элементов $M$ такая, что обогащение $(\mathfrak{M}, \bar{a})$ модели $\mathfrak{M}$ этими константами является простой моделью и семейство множеств атомов вычислимых булевых алгебр Линденбаума $F_{n}(\operatorname{Th}(\mathfrak{M}, \bar{a}))$ теории $\operatorname{Th}(\mathfrak{M}, \bar{a})$ над множеством формул от $n$ переменных равномерно вычислимо.

Е. А. Палютиным [24] был предложен пример счетно категоричной теории со следующими алгоритмическими свойствами. 
Теорема 2 (Е.А. Палютин [24]). Существует разрешимая счетно категоричная теория $T$ с элиминачией кванторов, для которой функиия $\alpha_{n}(T)$, равная мощности алгебры Линденбаума $F_{n}(T)$ для формул от $n$ переменных теории $T$, не общерекурсивна.

Функция $\alpha_{n}(T)$ называется функцией Рыль-Нардзевского счетно категоричной теории $T$. Из нерекурсивности функции Рыль-Нардзевского в теории Е. А. Палютина следует, что семейство полных формул этой теории не вычислимо. Из этих двух теорем мы получаем следующий результат.

СледствиЕ. Существует разрешимая счетно категоричная теория, счетная модель которой сильно конструктивизируема и неавтоустойчива относительно сильных конструктивизаций и даже неавтоустойчива.

В силу элиминации кванторов и разрешимости теории любая конструктивизация модели этой теории является сильной, а следовательно, она и неавтоустойчива.

Для разрешимых теорий всегда имеем, в силу теоремы Ю.Л. Ершова [4], сильно конструктивизируемую модель, но в случае эренфойхтовых теорий имеется более сильный результат.

Теорема 3 (М. Г. Перетятькин [25]). Простая модель эренфойхтовой разрешимой теории сильно конструктивизируема.

В общем случае был получен [33] следующий критерий разрешимости простых моделей.

Теорема 4 [33], [34]. Простая модель разрешимой теории сильно конструктивизируема, если и только если семейство главных типов этой теории вычислимо.

Приведем вначале конструкцию Е. А. Палютина [24] разрешимой счетно категоричной теории с элиминацией кванторов, которая будет встраиваться в теории при доказательстве основных теорем этой статьи.

\section{3. Теория Палютина}

Построим вначале вспомогательную теорию $T_{n}(S, R)$, где $n \in \mathbb{N}$.

Рассмотрим сигнатуру $\Sigma_{n}(S, T)$, состоящую из предикатов $S_{k}^{k+1}, R_{k}^{k+1}$ (где $k \leqslant n$, а $S_{k}$ и $R_{k}-(k+1)$-местные предикатные символы), а также предикатов $D_{k}^{2 k+2}$ местности $2 k+2$ для всех $k \leqslant n$.

Аксиомы теории $T_{n}(S, R)$ :

1. $S_{k}^{k+1}\left(x_{1}, \ldots, x_{k+1}\right) \rightarrow \neg x_{1}=x_{2}$ и $R_{k}^{k+1}\left(x_{1}, \ldots, x_{k+1}\right) \rightarrow \neg x_{1}=x_{2}$, если $1 \leqslant k<n$.

2. $S_{k}^{k+1}\left(x_{1}, \ldots, x_{k+1}\right) \leftrightarrow S_{k}^{k+1}\left(x_{\pi(1)}, \ldots, x_{\pi(k+1)}\right)$ и $R_{k}^{k+1}\left(x_{1}, \ldots, x_{k+1}\right) \leftrightarrow$ $R_{k}^{k+1}\left(x_{\pi(1)}, \ldots, x_{\pi(k+1)}\right)$, где $\pi$ - перестановка множества $\{1, \ldots, k+1\}, 1 \leqslant$ $k \leqslant n$.

3. $S_{k}^{k+1}\left(x_{1}, \ldots, x_{k+1}\right) \rightarrow \neg S_{l}^{l+1}\left(x_{i_{1}}, \ldots, x_{i_{l+1}}\right)$ и $R_{k}^{k+1}\left(x_{1}, \ldots, x_{k+1}\right) \rightarrow$ $\neg R_{l}^{l+1}\left(x_{i_{1}}, \ldots, x_{i_{l+1}}\right)$, если $l<k<n$ и $i_{1}<\cdots<i_{l+1}$. 
4. $R_{k}^{k+1}\left(x_{1}, \ldots, x_{k+1}\right) \rightarrow S_{k}^{k+1}\left(x_{1}, \ldots, x_{k+1}\right)$, если $k<n$.

5. Отношение $D_{n}\left(x_{1}, \ldots, x_{2 n+2}\right)$ определяет на множестве элементов, удовлетворяющих предикату $R_{0}^{n}\left(x_{1}, \ldots, x_{n+1}\right)$, отношение эквивалентности, состоящее в точности из двух классов, а на любом наборе элементов $x_{1}, \ldots, x_{2 n+2}$ таком, что не выполнено $R_{0}^{n}\left(x_{1}, \ldots, x_{n+1}\right)$ или не выполнено $R_{0}^{n}\left(x_{n+2}, \ldots, x_{2 n+2}\right)$, предикат $D_{n}^{k}\left(x_{1}, \ldots, x_{2 n+2}\right)$ ложен.

6. Отношение $R_{k}^{k+1}\left(x_{1}, \ldots, x_{k+1}\right)$ выполнено в точности на элементах одного из классов эквивалентности отношения $D_{k}\left(x_{1}, \ldots, x_{2 k+2}\right)$ в случае, если $k<$ $m, l<n$.

Конечная модель $\mathfrak{M}$ сигнатуры $\Sigma_{n}(S, T) \quad n$-допустимой, если она удовлетворяет аксиомам 1-5 и имеет основное множество $\{0,1, \ldots, r\}$.

Определим по любой допустимой конечной модели $\mathfrak{M}$ бескванторную формулу $\phi_{\mathfrak{M}}\left(x_{0}, \ldots, x_{r}\right)$ с переменными из $\left\{x_{0}, \ldots, x_{r}\right\}$, равную конъюнкции всех атомарных формул и их отрицаний, выполнимых на этой модели $\mathfrak{M}$ при подстановке вместо переменных $x_{0}, \ldots, x_{r}$ элементов $0, \ldots, r$.

7. Если $\mathfrak{M}-n$-допустимая модель из $r+1$ элемента, а $\mathfrak{N}-n$-допустимая модель из $r+2$ элементов и $\mathfrak{M} \subset \mathfrak{N}$, то

$$
\left(\forall x_{0}\right) \ldots\left(\forall x_{r}\right)\left(\phi_{\mathfrak{M}}\left(x_{0}, \ldots, x_{r}\right) \rightarrow\left(\exists x_{r+1}\right) \phi_{\mathfrak{N}}\left(x_{0}, \ldots, x_{r}, x_{r+1}\right)\right)
$$

Непротиворечивость $T_{n}(S, R)$ следует из справедливости свойства амальгамируемости для $n$-допустимых моделей: если $\mathfrak{M} \subseteq \mathfrak{N}_{1}$ и $\mathfrak{M} \subseteq \mathfrak{N}_{2}$, а $\mathfrak{M}, \mathfrak{N}_{1}$, $\mathfrak{N}_{2}-n$-допустимые модели, то модель $\mathfrak{N}_{1} \cup \mathfrak{N}_{2}$, где $N_{1} \cap N_{2}=M$, изоморфна $n$-допустимой модели. Нетрудно увидеть, что теория $T_{n}(S, R)$ счетно категорична, разрешима, допускает элиминацию кванторов, а функция $m \mapsto$ $\left|F_{m}\left(T_{n}(S, R)\right)\right|$, где $F_{m}\left(T_{n}(S, R)\right)$ - булева алгебра Линденбаума из формул со свободными переменными $x_{1}, \ldots, x_{m}$, рекурсивна.

Определим теперь теорию $T_{\mathbb{N}}=\bigcup_{n \geqslant 1} T_{n}(S, R)$.

Из вышесказанного следует, что эта теория разрешима и допускает элиминацию кванторов. Из элиминации кванторов и того, что предикаты $S_{k}$ и $K_{k}$ могут быть истинными только на различных элементах, легко вывести, в силу теоремы Рыль-Нардзевского [31], что построенная теория $T_{\mathbb{N}}$ счетно категорична.

Построенная теория является базовой для определимости в ней других теорий, из чего легко получить их счетную категоричность и элиминацию кванторов.

В конструкции искомой теории будем следовать примеру Палютина [24], в котором по любому рекурсивно перечислимому не рекурсивному множеству строится разрешимая счетно категоричная теория, у которой функция РыльНардзевского не рекурсивна и, следовательно, по критерию Нуртазина ее счетная модель сильно конструктивизируема, но не автоустойчива относительно сильных конструктивизаций. Заметим, что в счетно категоричной теории счетная модель является простой. 
Теорема 5. Существует разрешимая счетно категоричная теория с элиминачией кванторов, счетная модель которой не автоустойчива относительно сильных конструктивизаций и не автоустойчива, более того, класс ее конструктивизачий с точностью до рекурсивного изоморфизма не вычислим.

Приведем здесь некоторую простую модификацию теории Палютина [24], которая также определяет по любому рекурсивно перечислимому нерекурсивному множеству $Y$ счетно категоричную теорию $T_{Y}$.

Рассмотрим сигнатуру $\Sigma$, состоящую из предикатов $P_{n}^{k}$ и $D_{k}$ для $n \in \mathbb{N}$, $k \geqslant 1$ (где $P_{n}^{k}-(k+1)$-местный предикатный символ), а также отношений $D_{k}$ местности $2 k+2$ для каждого $k \geqslant 1$.

Пусть $f$ - рекурсивная функция, перечисляющая множество $Y$ без повторений, а $\tilde{f}$ - рекурсивная функция, определенная следующим образом:

$$
\tilde{f}(x, y)= \begin{cases}0, & \text { если } x<y \text { и нет } r<y \text { такого, что } g(r)=x, \\ 1 & \text { в противном случае. }\end{cases}
$$

Полагаем $n \stackrel{k}{\sim} m$, если $\tilde{f}(k, n)=\tilde{f}(k, m)$.

Рассмотрим аппроксимацию $\Sigma_{m}, m \geqslant 1$, этой сигнатуры $\Sigma$, где $\Sigma_{m}$ состоит из предикатных символов $P_{n}^{k}, n \leqslant m, 0 \leqslant k \leqslant m$, и отношений $D_{n}^{2 n+2}$ местности $2 n+2$ для каждого $n \leqslant m$. Определим в сигнатуре $\Sigma_{m}$ теорию $T_{m}^{Y}$.

Аксиомы теории $T_{m}^{Y}$ :

1. $P_{n}^{k}\left(x_{1}, \ldots, x_{k+1}\right) \rightarrow \neg x_{1}=x_{2}$, если $0 \leqslant k \leqslant m, n \leqslant m$.

2. $P_{n}^{k}\left(x_{1}, \ldots, x_{k+1}\right) \leftrightarrow P_{n}^{k+1}\left(x_{\pi(1)}, \ldots, x_{\pi(k+1)}\right)$, где $\pi$ перестановка множества $\{1, \ldots, k+1\}, 0 \leqslant k \leqslant m, n \leqslant m$.

3. $P_{n}^{k}\left(x_{1}, \ldots, x_{k+1}\right) \leftrightarrow \neg P_{r}^{l}\left(x_{i_{1}}, \ldots, x_{i_{l+1}}\right)$, если $k \leqslant m, n \leqslant m, r \leqslant m, l<k$, $r \neq n$ и $i_{1}<\cdots<i_{l+1}$.

4. $P_{n}^{k}\left(x_{1}, \ldots, x_{k+1}\right) \rightarrow P_{l}^{k}\left(x_{1}, \ldots, x_{k+1}\right)$, если $k \leqslant m, n \leqslant m, l<n$ и $\neg n \stackrel{k}{\sim} l$, и $P_{l}^{k}\left(x_{1}, \ldots, x_{k+1}\right) \rightarrow P_{n}^{k}\left(x_{1}, \ldots, x_{k+1}\right)$, если $k \leqslant m, n \leqslant m, l<n$ и $n \stackrel{k}{\sim} l$.

5. Отношение $D_{n}\left(x_{1}, \ldots, x_{n+1}, y_{1}, \ldots, y_{n+1}\right)$ определяет на множестве элементов, удовлетворяющих предикату $P_{0}^{n}\left(x_{1}, \ldots, x_{n+1}\right)$, отношение эквивалентности, состоящее из не более чем двух классов, а на любом наборе элементов $x_{1}, \ldots, x_{n+1}, y_{1}, \ldots, y_{n+1}$ таком, что не выполнено $P_{0}^{n}\left(x_{1}, \ldots, x_{n+1}\right)$ или не выполнено $P_{0}^{n}\left(y_{1}, \ldots, y_{n+1}\right)$, предикат $D_{n}^{k}\left(x_{1}, \ldots, x_{n+1}, y_{1}, \ldots, y_{n+1}\right)$ ложен, отношение $P_{l}^{k}\left(x_{1}, \ldots, x_{k+1}\right)$ выполнено в точности на элементах одного из классов эквивалентности отношения $D_{k}\left(x_{1}, \ldots, x_{2 k+2}\right)$, если $k \leqslant m, l<n$ и $\neg 0 \stackrel{k}{\sim} l$.

Конечная модель $\mathfrak{M}$ сигнатуры $\Sigma_{m}$ называется $m$-допустимой, если она удовлетворяет аксиомам $1-5$ и имеет основное множество $\{0,1, \ldots, r\}$.

Определим по любой допустимой конечной модели $\mathfrak{M}$ бескванторную формулу $\phi_{\mathfrak{M}}\left(x_{0}, \ldots, x_{r}\right)$ с переменными из $\left\{x_{0}, \ldots, x_{r}\right\}$, равную конъюнкции всех атомарных формул и их отрицаний, выполнимых на этой модели $\mathfrak{M}$ при подстановке вместо переменных $x_{0}, \ldots, x_{r}$ элементов $0, \ldots, r$.

6 . Если $\mathfrak{M}-m$-допустимая модель из $r+1$ элемента, а $\mathfrak{N}-m$-допустимая модель из $r+2$ элементов и $\mathfrak{M} \subset \mathfrak{N}$, то

$$
\left(\forall x_{0}\right) \ldots\left(\forall x_{r}\right)\left(\phi_{\mathfrak{M}}\left(x_{0}, \ldots, x_{r}\right) \rightarrow\left(\exists x_{r+1}\right) \phi_{\mathfrak{N}}\left(x_{0}, \ldots, x_{r}, x_{r+1}\right)\right) .
$$


Непротиворечивость $T_{m}^{Y}$ следует из справедливости свойства амальгамируемости для $m$-допустимых моделей, т. е. если $\mathfrak{M} \subseteq \mathfrak{N}_{1}$ и $\mathfrak{M} \subseteq \mathfrak{N}_{2}$, а $\mathfrak{M}, \mathfrak{N}_{1}, \mathfrak{N}_{2}$ $m$-допустимые модели, то модель $\mathfrak{N}_{1} \cup \mathfrak{N}_{2}$, где $N_{1} \cap N_{2}=M$, изоморфна $m$-допустимой модели. Нетрудно увидеть, что теория $T_{m}^{Y}$ счетно категорична, разрешима, допускает элиминацию кванторов, а функция $n \mapsto\left|F_{n}\left(T_{m}^{Y}\right)\right|$, где $F_{n}\left(T_{m}^{Y}\right)$ - булева алгебра Линденбаума из формул со свободными переменными $x_{1}, \ldots, x_{n}$, рекурсивна.

Определим теперь теорию $T_{Y}=\bigcup_{m \geqslant 1} T_{m}^{Y}$.

Из вышесказанного следует, что эта теория разрешима и допускает элиминацию кванторов. Из элиминации кванторов и того, что предикат $P_{n}^{k}$ истинен только на $k$ различных элементах, а предикаты $P_{n}^{k}$ для разных $n$ либо все эквивалентны - если $k \notin Y$, либо если $g(m)=k$, то $P_{0}^{k}, \ldots, P_{m^{\prime}-1}^{k}$ эквивалентны и $P_{m^{\prime}}^{k}, P_{m+s}^{k}$ также эквивалентны для любого $s \geqslant 0$, где $m^{\prime}=\max \{m, n\}+1$, следует в силу теоремы Рыль-Нардзевского [31], что построенная теория счетно категорична.

Заметим, что в теории $T_{Y}$ уже функция $n \mapsto\left|F_{n}\left(T_{Y}\right)\right|$ не рекурсивна [24], если множество $Y$ не рекурсивно.

Если бы она была рекурсивна, то мы нашли бы все попарно неэквивалентные бескванторные формулы из $F_{n+1}\left(T_{Y}\right)$ и отсюда узнали для $P_{m}^{n+1}, m \in \mathbb{N}$, все ли $P_{m}^{n+1}$ эквивалентны, но тогда $Y$ было бы рекурсивным множеством.

Отсюда получаем, что множество полных формул в теории $T_{Y}$ не вычислимо. А тогда по теореме Ершова ее счетная модель разрешима, а по теореме Нуртазина ее счетная модель проста и неавтоустойчива относительно сильных конструктивизаций, а в силу элиминации кванторов всякая ее конструктивизация является сильной, и поэтому класс ее конструктивизаций не вычислим и она неавтоустойчива.

\section{4. Эренфойхтова теория с автоустойчивой простой моделью}

В этом разделе мы изучим вопрос о наследовании автоустойчивости относительно сильных конструктивизаций в простых относительно обогащения константами моделях. Будем следовать работе [35].

ТЕОРема 6. Существует разрешимая эренфойхтова теория, простая модель которой автоустойчива относительно сильных конструктивизаций и даже автоустойчива, но некоторая модель которой, простая в конечном обогащении константами, сильно конструктивизируема, но не автоустойчива.

Перейдем к доказательству теоремы.

Определим для $m \geqslant 1$ сигнатуру $\Sigma_{Y}^{m}$, состоящую из одноместных предикатов $A, B$, двухместного отношения $\leqslant$, двухместного предиката $F$, одноместных предикатов $\mathscr{D}_{n}, n \leqslant m$, и $k$-местных предикатов $P_{n}^{k}$ для $n \leqslant m, 1 \leqslant k \leqslant m$, а также бинарного предикатного символа $\leqslant{ }^{*}$ и одноместных предикатов $\mathscr{D}_{n}^{*}$, $n \leqslant m$.

Определим аксиомы нашей теории $\widehat{T}_{Y}^{m}$ для рекурсивно перечислимого не рекурсивного множества $Y$. 
1. $(\forall x)(A(x) \vee B(x))$.

2. $\neg(\exists x)(A(x) \& B(x))$.

3. Отношение $\leqslant$ задает на $A$ линейный порядок.

4. Отношение $\leqslant$ задает плотный линейный порядок без концов на $A$.

5. $\mathscr{D}_{n}$ для $n \leqslant m$ является начальным сегментом в $(A, \leqslant)$.

6. $\mathscr{D}_{n}$ не имеет точной верхней грани.

7. $\mathscr{D}_{n} \subset \mathscr{D}_{n+1}$.

8. Отношение $F$ является подмножеством в $B \times A$ и $(\forall x)\left(\forall x^{\prime}\right)(\forall y)((F(y, x) \&$ $\left.\left.F\left(y, x^{\prime}\right)\right) \rightarrow x=x^{\prime}\right)$.

9. Отношение $F$ является графиком отображения из $B$ на $A$ такого, что

$$
(\forall x)(A(x) \rightarrow(\exists y) F(y, x)) \&(\forall y)(B(y) \rightarrow(\exists x) F(y, x))
$$

10. Каждый элемент из $A$ имеет бесконечно много прообразов в $B$, т. е. $(\forall x)\left(A(x) \rightarrow\left(\exists x_{1}, \ldots, \exists x_{n}\right)\left(\bigwedge_{i} F\left(x_{i}, x\right) \& \bigwedge_{i<j} \neg x_{i}=x_{j}\right)\right)$ для $n \geqslant 1$ и $n \in \mathbb{N}$.

11. $\left(F\left(x, x^{\prime}\right) \& F\left(y, y^{\prime}\right) \& x^{\prime} \leqslant y^{\prime} \&(B(x) \& B(y))\right) \vee\left(F\left(x, x^{\prime}\right) \& x^{\prime} \leqslant y \&\right.$ $(B(x) \& A(y))) \vee\left(F\left(y, y^{\prime}\right) \& x \leqslant y^{\prime} \&(A(x) \& B(y))\right) \vee(x \leqslant y \&(A(x) \& A(y))) \rightarrow$ $x \leqslant * y$.

12. $x \leqslant *^{*} y \rightarrow\left(\left(\exists x^{\prime}\right)\left(\exists y^{\prime}\right)\left(F\left(x, x^{\prime}\right) \& F\left(y, y^{\prime}\right) \& x^{\prime} \leqslant y^{\prime} \&(B(x) \& B(y))\right) \vee\right.$ $\left(\exists x^{\prime}\right)\left(F\left(x, x^{\prime}\right) \& x^{\prime} \leqslant y \&(B(x) \& A(y))\right) \vee\left(\exists y^{\prime}\right)\left(F\left(y, y^{\prime}\right) \& x \leqslant y^{\prime} \&\right.$ $(A(x) \& B(y))) \vee(x \leqslant y \&(A(x) \& A(y))))$.

13. $\mathscr{D}_{n}^{*}(x) \rightarrow\left(\exists x^{\prime}\right)\left(F\left(x, x^{\prime}\right) \& \mathscr{D}_{n}\left(x^{\prime}\right)\right)$.

14. $\left(F\left(x, x^{\prime}\right) \& \mathscr{D}_{n}\left(x^{\prime}\right)\right) \rightarrow \mathscr{D}_{n}^{*}(x)$.

Аксиомы для предикатов $P_{n}^{k}$ при $n \leqslant m, k \leqslant m$ :

P1. $P_{n}^{k+1}\left(x_{1}, \ldots, x_{k+1}\right) \rightarrow \neg x_{1}=x_{2}$.

P2. $P_{n}^{k+1}\left(x_{1}, \ldots, x_{k+1}\right) \rightarrow P_{n}^{k+1}\left(x_{\pi(1)}, \ldots, x_{\pi(k+1)}\right)$, где $\pi$ - перестановка элементов $\{1, \ldots, k+1\}$.

P3. $P_{n}^{k+1}\left(x_{1}, \ldots, x_{k+1}\right) \rightarrow(\exists y)\left(\bigwedge_{i=1}^{k+1} F\left(x_{i}, y\right)\right)$.

P4. $\left(P_{n}^{k+1}\left(x_{1}, \ldots, x_{k+1}\right) \& F\left(x_{1}, y\right)\right) \rightarrow \neg \mathscr{D}_{s}(y)$ для $s \leqslant m$ и $(s<n$ или $s<k)$, a также $\left(P_{n}^{k+1}\left(x_{1}, \ldots, x_{k+1}\right) \& F\left(x_{1}, y\right) \& F\left(x_{1}, y^{\prime}\right)\right) \rightarrow \neg y=y^{\prime}$.

P5. $P_{n}^{k+1}\left(x_{1}, \ldots, x_{k+1}\right) \leftrightarrow P_{l}^{k+1}\left(x_{1}, \ldots, x_{k+1}\right)$, если $k<m, n<m, l<m$

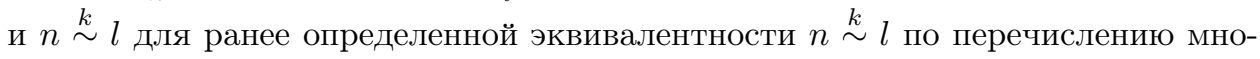
жества $Y$.

Конечная модель $\mathfrak{M}$ называется $m$-допустимой, если удовлетворяет аксиомам $1-3,5,7,8,11,14$ для $n \leqslant m$ и аксиомам 9,10 и $\mathrm{P} 1, \mathrm{P} 2, \mathrm{P} 4, \mathrm{P} 5$, а основное множество равно $\{0, \ldots, r\}$.

Определим по $\mathfrak{M}$ бескванторную формулу $\phi_{\mathfrak{M}}$, равную конъюнкции всех атомарных формул с переменными из $\left\{x_{0}, \ldots, x_{r}\right\}$, истинную при подстановке элементов $0, \ldots, r$ в $\mathfrak{M}$.

Р6. Пусть $\mathfrak{M}, \mathfrak{N}-m$-допустимые модели с основными множествами $\{0, \ldots, r\}$ и $\{0, \ldots, r, r+s\}$ соответственно. Пусть $\mathfrak{M} \subset \mathfrak{N}$. Тогда включим в нашу теорию 
аксиому

$$
\begin{aligned}
\left(\forall x_{0}\right) \ldots\left(\forall x_{r}\right) & \left(\exists x_{r+1}\right) \ldots\left(\exists x_{r+s}\right)\left(\phi_{\mathfrak{M}}\left(x_{0}, \ldots, x_{r}\right)\right. \\
& \left.\rightarrow \phi_{\mathfrak{N}}\left(x_{0}, \ldots, x_{r}, x_{r+1}, \ldots, x_{r+s}\right)\right) .
\end{aligned}
$$

Заметим, что теория $\widehat{T}_{Y}^{m}$ счетно категорична, допускает элиминацию кванторов и разрешима, а множество полных формул у нее вычислимо равномерно по $m$.

Непротиворечивость легко получить непосредственным построением модели этой теории из модели теории Палютина.

Доказательство счетной категоричности следует из простой проверки изоморфизма любых двух счетных моделей этой теории челночным методом построения изоморфизма.

Из счетной категоричности и бесконечности ее моделей следует полнота и разрешимость этой теории. Отсюда и из конечности сигнатуры и элиминации кванторов сразу получаем вычислимость множества ее полных формул.

Определим теперь теорию $\widehat{T}_{Y}^{*}=\bigcup \widehat{T}_{Y}^{m}$. Из свойств теории $\widehat{T}_{Y}^{m}$ получаем полноту, разрешимость и элиминацию кванторов для теории $\widehat{T}_{Y}^{*}$.

Рассмотрим три модели этой теории: $\mathfrak{M}_{1}$, в которой $\bigcup_{i \geqslant 0} \mathscr{D}_{i}=A ; \mathfrak{M}_{2}$, в которой подмножество $A \backslash\left(\bigcup_{i \geqslant 0} \mathscr{D}_{i}\right)$ имеет наименьший элемент; $\mathfrak{M}_{3}$, у которой $\varnothing \neq A \backslash\left(\bigcup_{i \geqslant 0} \mathscr{D}_{i}\right)$ и в $A \backslash\left(\bigcup_{i \geqslant 0} \mathscr{D}_{i}\right)$ нет наименьшего элемента.

Пусть $\mathfrak{M}$ - модель теории $\widehat{T}_{Y}^{*}, a_{1}, a_{2} \in \mathfrak{M}$. Из счетной категоричности теории $\widehat{T}_{Y}^{m}$ получаем, что для любых элементов $a_{1}, a_{2} \in \mathscr{D}_{i+1} \backslash \mathscr{D}_{i}$ подмодели $\left\{b \mid F(b)=a_{i}\right\}, i \in\{1,2\}$, сигнатуры $\left\langle P_{n}^{k+1} \mid n \in \mathbb{N}, k \geqslant 1\right\rangle$ изоморфны.

Из счетной категоричности теории Палютина для любых элементов $a_{1}, a_{2} \in$ $A^{\prime} \cup \mathscr{D}_{i}$, где $A^{\prime}=A \backslash\left(\bigcup_{i \geqslant 0} \mathscr{D}_{i}\right)$, следует, что подмодели $\left\{b \mid F(b)=a_{i}\right\}, i \in\{1,2\}$, сигнатуры $\left\langle P_{n}^{k+1} \mid n \in \mathbb{N}, k \geqslant 1\right\rangle$ изоморфны.

Отсюда непосредственным построением изоморфизма на основе плотности порядка $\leqslant$ на $A$ получаем, что любая счетная модель $\mathfrak{M}$ теории $\widehat{T}_{Y}$ изоморфна одной из моделей $\mathfrak{M}_{1}, \mathfrak{M}_{2}, \mathfrak{M}_{3}$, а модели $\mathfrak{M}_{1}, \mathfrak{M}_{2}, \mathfrak{M}_{3}$ попарно не изоморфны, т. е. теория $\widehat{T}_{Y}$ эренфойхтова с тремя моделями.

Для моделей $\mathfrak{M}_{2}$ и $\mathfrak{M}_{3}$ легко построить их конструктивизации, а из элиминации кванторов и разрешимости теории $\widehat{T}_{Y}$ получаем, что любая ее конструктивизация является сильной, а простая сильно конструктивизируема и в силу элиминации кванторов любая ее конструктивизация сильная. Итак, получаем, что если $\mathfrak{M}$ - счетная модель теории $\widehat{T}_{Y}$, то она сильно конструктивизируема и любая ее конструктивизация является сильной.

Заметим, что простая модель $\mathfrak{M}_{1}$ теории $\widehat{T}_{Y}$ автоустойчива относительно сильных конструктивизаций и, следовательно, автоустойчива.

Доказательство следует из сильной конструктивизируемости и того факта, что для любого $a \in \mathscr{D}_{i}$ подмодель $\{b \mid F(b)=a\}$ имеет вычислимое множество 
полных формул, что позволяет проводить построение эффективно. Существование сильной конструктивизации следует из теоремы Перетятькина о разрешимости простых моделей разрешимых эренфойхтовых теорий.

Покажем, что если модель $\mathfrak{M}$ теории $\widehat{T}_{Y}$ не проста, то она не автоустойчива (относительно сильных конструктивизаций).

Для доказательства рассмотрим любой элемент $a \in A \backslash\left(\bigcup_{i \geqslant 0} \mathscr{D}_{i}\right)$ в модели $\mathfrak{M}_{i}, i \in\{2,3\}$. В этом случае по теореме Нуртазина 1 в автоустойчивой модели относительно сильных конструктивизаций существует набор элементов $\bar{a}$ такой, что $(\mathfrak{M}, \bar{a})$ - простая модель ее теории и множество полных формул вычислимо. Так как любая конструктивизация $\mathfrak{M}$ в силу разрешимости $\widehat{T}_{Y}$ и элиминации кванторов является сильной, то автоустойчивость эквивалентна автоустойчивости относительно сильных конструктивизаций. Заметим, что насыщенная модель для любого обогащения $(\mathfrak{M}, \bar{a})$ не проста. В случае $\mathfrak{M} \cong \mathfrak{M}_{2}$ рассмотрим элемент $a$, являющийся наименьшим в $A \backslash\left(\bigcup_{i \geqslant 0} \mathscr{D}_{i}\right)$. Отсюда легко следует, что и модель $(\mathfrak{M}, \bar{a}, a)$ проста и имеет вычислимое множество полных формул. Заметим, что $n \notin Y$, если и только если для всех $m$ формулы

$$
\bigwedge_{i=1}^{n+1} F\left(x_{i}\right)=a \& P_{m}^{n+1}\left(x_{1}, \ldots, x_{n}\right)
$$

эквивалентны в нашей теории.

Но подмодель $\{b \mid F(b)=a\}$ в модели $\mathfrak{M}$, ограниченная на сигнатуру $\left\langle P_{m}^{n+1}, m \in \mathbb{N}, n \in \mathbb{N}\right\rangle$, будет моделью теории Палютина $T_{Y}$, а она счетно категорична, но имеет невычислимое множество полных формул. Так как от $n+1$ переменной имеется лишь конечное число попарно неэквивалентных формул, мы, зная их всех, можем решить и вопрос об эквивалентности всех формул $P_{m}^{n+1}$ в ней, что противоречит нерекурсивности множества $Y$. Почти простота модели $\mathfrak{M}_{2}$ следует из того, что обогащение наименьшим элементом $a \in A \backslash\left(\bigcup_{i \geqslant 0} \mathscr{D}_{i}\right)$ уже дает простоту этого обогащения.

Теорема 6 доказана.

\section{5. Теории с неавтоустойчивой простой моделью}

В этом разделе мы изучим вопрос о наследовании неавтоустойчивости в простых обогащенных константами моделях.

Вначале приведем новое доказательство теоремы К. Ж. Кудайбергенова [36], в которой строится теория с несчетным множеством типов и счетных моделей, у которой простая модель неавтоустойчива относительно сильных конструктивизаций, а некоторая почти простая модель автоустойчива и имеет сильную конструктивизацию.

Теорема 7 [36]. Существует полная разрешимая теория с несчетным множеством типов, простая модель которой сильно конструктивизируема 
и неавтоустойчива относительно сильных конструктивизаций, но для которой существует простая модель в конечном обогащении константами, которая сильно конструктивизируема и автоустойчива относительно сильных конструктивизачий.

ДокАзАтЕльство. Как и в доказательстве предыдущей теоремы, рассмотрим рекурсивно перечислимое, но не рекурсивное множество $Y$. Пусть $f$ : $\mathbb{N} \underset{\text { на }}{\stackrel{1-1}{\rightarrow}} Y$ - рекурсивная функция, перечисляющая это множество без повторений. Как и ранее, пишем $n \stackrel{k}{\sim} \tilde{f} m$, если $\tilde{f}(k, n)=\tilde{f}(k, m)$, где

$$
\tilde{f}(k, m)= \begin{cases}0, & \text { если } k<m \text { и нет } r<m \text { такого, что } f(r)=k, \\ 1 & \text { в противном случае. }\end{cases}
$$

Построим равномерно по $m$ разрешимую счетно категоричную с элиминацией кванторов теорию $T(m, Y)$ с вычислимым множеством полных формул. Определим теперь для $m \geqslant 1$ сигнатуру $\Sigma_{Y}^{m}$, состоящую из одноместных предикатов $A, B$, двухместного отношения $\leqslant$, одноместных отношений $\mathscr{D}_{n}, \Delta_{n}$ для $n \leqslant m$, предикатов $P_{n}^{k+1}$ местности $k+1$ для $0 \leqslant k \leqslant m$ и $n \leqslant m$, предикатов $Q_{k}^{k+1}$ местности $k+2$ для $0 \leqslant k \leqslant m$, двухместных предикатов $R_{k}$ и $(2 k+2)$-местных отношений $R_{k}^{*}$ для $k \leqslant m$.

Определим аксиомы нашей теории $T(m, Y)$ для рекурсивно перечислимого не рекурсивного множества $Y$.

1. $(\forall x)(A(x) \vee B(x))$.

2. $\neg(\exists x)(A(x) \& B(x))$.

3. Отношение $\leqslant$ задает на $A$ линейный порядок.

4. Отношение $\leqslant$ задает плотный линейный порядок без концов на $A$.

5. $\mathscr{D}_{n}$ для $n \leqslant m$ является начальным сегментом в $(A, \leqslant)$.

6. $\mathscr{D}_{n}$ для $n \leqslant m$ является начальным сегментом в $(A, \leqslant)$ без точной верхней грани, а тогда и $\neg \mathscr{D}_{n}$ для $n \leqslant m$ не имеет наименьшего элемента.

7. $\mathscr{D}_{n} \subseteq \mathscr{D}_{n+1}$ для $n \leqslant m$.

8. Формульные множества $\mathscr{D}_{n}$ и $\mathscr{D}_{n+1}$ различны.

9. $\Delta_{n+1} \subseteq \Delta_{n}$ для $n<m$.

10. $\Delta_{n+1} \subset\left(\Delta_{n+1} \backslash \mathscr{D}_{n+1}\right)$.

11. Формульные множества $\Delta_{n}$ и $\Delta_{n+1}$ различны для $n<m$.

12. $\Delta_{n+1}-$ собственное подмножество $\Delta_{n}$.

13. Множества $\Delta_{n}$ и $A \backslash\left(\mathscr{D}_{n} \cup \Delta_{n}\right)$ - плотные подмножества в $A \backslash \mathscr{D}_{n}$ относительно порядка $(A, \leqslant)$ для $n<m$.

14. Множество $\Delta_{n} \& \neg \Delta_{n+1}-$ плотное подмножество в $\Delta_{n} \backslash \mathscr{D}_{n+1}$ относительно порядка $(A, \leqslant)$ для $n<m$.

Аксиомы для предикатов $P_{n}^{k}$ при $n \leqslant m, k \leqslant m$.

P1. $P_{n}^{k+1}\left(x_{1}, \ldots, x_{k+1}\right) \rightarrow \neg x_{1}=x_{2}$ для $n \leqslant m, k \leqslant m$.

P2. $P_{n}^{k+1}\left(x_{1}, \ldots, x_{k+1}\right) \rightarrow P_{n}^{k+1}\left(x_{\pi(1)}, \ldots, x_{\pi(k+1)}\right)$, где $\pi$ - перестановка элементов $\{1, \ldots, k+1\}$ для $n \leqslant m, k \leqslant m$.

P3. $P_{n}^{k+1}\left(x_{1}, \ldots, x_{k+1}\right) \leftrightarrow P_{l}^{k+1}\left(x_{1}, \ldots, x_{k+1}\right)$, если $k \leqslant m, n \leqslant m, l \leqslant m$ и $n \stackrel{k}{\sim} l$ для ранее определенной эквивалентности $n \stackrel{k}{\sim} l$ по перечислению множества $Y$. 
P4. $P_{n}^{k+1}\left(x_{1}, \ldots, x_{k+1}\right) \rightarrow\left(B\left(x_{1}\right) \& \cdots \& B\left(x_{k+1}\right)\right)$ для $n \leqslant m, k \leqslant m$.

Q1. $Q_{k}^{k+1}\left(z, x_{1}, \ldots, x_{k+1}\right) \rightarrow\left(\neg x_{1}=x_{2} \& \Delta_{k}(z) \& \neg \mathscr{D}_{k}(z)\right)$ для $k \leqslant m$.

Q2. $Q_{k}^{k+1}\left(z, x_{1}, \ldots, x_{k+1}\right) \rightarrow Q_{k}^{k+1}\left(z, x_{\pi(1)}, \ldots, x_{\pi(k+1)}\right)$, где $\pi$ - перестановка элементов $\{1, \ldots, k+1\}$ и $k \leqslant m$.

Q3. $(\exists z)\left(\forall x_{1}\right) \ldots\left(\forall x_{k+1}\right)\left(P_{l}^{k+1}\left(x_{1}, \ldots, x_{k+1}\right) \leftrightarrow Q_{k}^{k+1}\left(z, x_{1}, \ldots, x_{k+1}\right)\right)$, если $k<m, n<m, l<m$ и $\neg 0 \stackrel{k}{\sim} l$ для ранее определенной эквивалентности $n \stackrel{k}{\sim} l$ по перечислению множества $Y$.

Q4. $\left(\forall z_{1}\right)\left(\left(\forall z_{2}\right)\left(\left(\Delta_{k}\left(z_{1}\right) \& \Delta_{k}\left(z_{2}\right)\right) \rightarrow\left(\left(\forall x_{1}\right) \ldots\left(\forall x_{k+1}\right)\right)\left(\left(\left(\underset{i=0}{\&} B\left(x_{i}\right)\right) \&\right.\right.\right.\right.$ $\left.\left(\underset{1 \leqslant i<j \leqslant k+1}{\&} \neg x_{i}=x_{j}\right)\right) \rightarrow\left(Q_{k}^{k+1}\left(z_{1}, x_{1}, \ldots, x_{k+1}\right) \leftrightarrow Q_{k}^{k+1}\left(z_{2}, x_{1}\right.\right.$,

$\left.\left.\left.\ldots, x_{k+1}\right)\right)\right) \vee\left(\forall x_{1}\right) \ldots\left(\forall x_{k+1}\right)\left(\left(\left(\underset{i=0}{\&} B\left(x_{i}\right)\right) \&\left(\underset{1 \leqslant i<j \leqslant k+1}{\&} \neg x_{i}=x_{j}\right)\right) \rightarrow\right.$ $\left.\left.\left.\left(Q_{k}^{k+1}\left(z_{1}, x_{1}, \ldots, x_{k+1}\right) \leftrightarrow \neg Q_{k}^{k+1}\left(z_{2}, x_{1}, \ldots, x_{k+1}\right)\right)\right)\right)\right)$ для $k \leqslant m$.

Q5. $\left(\forall z_{1}\right)\left(\forall z_{2}\right)\left(\left(\left(z_{1}<z_{2}\right) \& \Delta_{k}\left(z_{1}\right) \& \Delta_{k}\left(z_{2}\right)\right) \rightarrow \underset{0 \leqslant \varepsilon_{0}, \ldots, \varepsilon_{k} \leqslant 1}{\&}\left(\exists u_{1}\right)\left(\exists u_{2}\right)\left(\exists u_{3}\right)\right.$ $\left(\left(\left(u_{1}<z_{1}\right) \&\left(z_{1}<u_{2}<z_{2}\right) \&\left(z_{2}<u_{3}\right)\right) \& \stackrel{s=0}{3}_{s=0}\left(\left(\Delta_{k}\left(u_{s}\right) \& \neg \Delta_{k+1}\left(u_{s}\right)\right)\right.\right.$ $\& \underset{i=0}{\&}\left(\left(\forall x_{1}\right) \ldots\left(\forall x_{i+1}\right)\left(\left(\underset{j=0}{i} B\left(x_{j}\right)\right) \&\left(\underset{1 \leqslant r<q \leqslant k+1}{\&} \neg x_{r}=x_{q}\right)\right) \rightarrow\right.$ $\left.\left.\left.\left.\left(Q_{i}^{i+1}\left(z_{1}, x_{1}, \ldots, x_{i+1}\right) \leftrightarrow\left(Q_{i}^{i+1}\right)^{\varepsilon_{i}}\left(u_{s}, x_{1}, \ldots, x_{i+1}\right)\right)\right)\right)\right)\right)$ для $k<m$ и

$\left(\forall z_{1}\right)\left(\left(\forall z_{2}\right)\left(z_{1}<z_{2} \& \Delta_{m}\left(z_{1}\right) \& \Delta_{m}\left(z_{2}\right)\right) \rightarrow\right.$

$\underset{\varepsilon_{0}, \ldots, \varepsilon_{m}}{\&}\left(\exists u_{1}\right)\left(\exists u_{2}\right)\left(\exists u_{3}\right)\left(\left(u_{1}<z_{1} \&\left(z_{1}<u_{2}<z_{2}\right) \& z_{2}<u_{3}\right)\right.$

$\& \underset{s=0}{\stackrel{3}{\&}}\left(\Delta_{m}\left(u_{s}\right) \& \underset{i=0}{\stackrel{m}{\&}}\left(\left(\forall x_{1}\right) \ldots\left(\forall x_{i+1}\right)\left(\left(\underset{j=0}{\stackrel{i}{\&} B} B\left(x_{j}\right)\right) \&\left(\underset{1 \leqslant r<q \leqslant k+1}{\&} \neg x_{r}=x_{q}\right)\right) \rightarrow\right.\right.$ $\left.\left.\left.\left.\left(Q_{i}^{i+1}\left(z_{1}, x_{1}, \ldots, x_{i+1}\right) \leftrightarrow\left(Q_{i}^{i+1}\right)^{\varepsilon_{i}}\left(u_{s}, x_{1}, \ldots, x_{i+1}\right)\right)\right)\right)\right)\right)$.

Q6. $Q_{k}^{k+1}\left(z, x_{1}, \ldots, x_{k+1}\right) \rightarrow\left(\left(B\left(x_{1}\right) \& \cdots \& B\left(x_{k+1}\right)\right) \& \Delta_{k}(z)\right)$.

Q7. Отношения эквивалентности $R_{n}(x, y)$ на $\Delta_{n}, 0 \leqslant n \leqslant m$, таковы, что выполняется аксиома

$$
\begin{aligned}
{\left[R_{k}(x, y)\right.} & \left.\rightarrow \Delta_{k}(x) \& \Delta_{k}(y)\right] \&\left[R_{k}(x, y)\right. \\
& \leftrightarrow\left(\forall x_{1}\right) \ldots\left(\forall x_{k+1}\right)\left(Q_{k}^{k+1}\left(x, x_{1}, \ldots, x_{k+1}\right)\right. \\
& \left.\left.\leftrightarrow Q_{k}^{k+1}\left(y, x_{1}, \ldots, x_{k+1}\right)\right) \&\left(\exists x_{1}\right) \ldots\left(\exists x_{k+1}\right) Q_{k}^{k+1}\left(x, x_{1}, \ldots, x_{k+1}\right)\right] .
\end{aligned}
$$

Q8. $R_{k}^{*}\left(x_{1}, \ldots, x_{k+1}, y_{1}, \ldots, y_{k+1}\right) \leftrightarrow(\exists x)\left(Q_{k}^{k+1}\left(x, x_{1}, \ldots, x_{k+1}\right) \& Q_{k}^{k+1}\left(x, y_{1}\right.\right.$, $\left.\left.\ldots, y_{k+1}\right)\right)$.

Q9. $\left(\forall x_{1}\right) \ldots\left(\forall x_{k+1}\right)\left(P_{0}^{k+1}\left(x_{1}, \ldots, x_{k+1}\right) \rightarrow\left(\exists z_{1}\right)\left(\exists z_{2}\right)\left(Q_{k}^{k+1}\left(z_{1}, x_{1}, \ldots, x_{k+1}\right)\right.\right.$ $\left.\left.\& \neg Q_{k}^{k+1}\left(z_{1}, x_{1}, \ldots, x_{k+1}\right)\right)\right)$.

Q10. $R_{k}^{*}$ - отношение эквивалентности из двух классов на множестве наборов из попарно различных элементов из $B, k \leqslant m$.

Q11. $R_{k}$ - отношение эквивалентности из двух классов на $\Delta_{k}$.

Q12. Оба класса отношения $R_{k}$ плотные подмножества в $\Delta_{k}$. 
Конечная модель $\mathfrak{M}$ называется $m$-допустимой, если она удовлетворяет аксиомам 1-3, 5, 7-12, P1-P4, Q1-Q4 и Q6-Q11, а основное множество равно $\{0, \ldots, r\}$. Обозначим эту теорию $T_{0}^{m}$.

Определим по $\mathfrak{M}$ бескванторную формулу $\phi_{\mathfrak{M}}$, равную конъюнкции всех атомарных формул с переменными из $\left\{x_{0}, \ldots, x_{r}\right\}$, истинную при подстановке элементов $0, \ldots, r$ в $\mathfrak{M}$.

Аксиома плотности. Пусть $\mathfrak{M}, \mathfrak{N}-m$-допустимые модели с основными множествами $\{0, \ldots, r\}$ и $\{0, \ldots, r, r+s\}$ соответственно. Пусть $\mathfrak{M} \subset \mathfrak{N}$. Тогда включим в нашу теорию аксиому

$$
\left(\forall x_{0}, \ldots, x_{r}\right)\left(\phi_{\mathfrak{M}}\left(x_{0}, \ldots, x_{r}\right) \leftrightarrow\left(\exists x_{r+1}, \ldots, x_{r+s}\right) \phi_{\mathfrak{N}}\left(x_{0}, \ldots, x_{r}, x_{r+1}, \ldots, x_{r+s}\right)\right) .
$$

Лемма 1. Теория $T(m, Y)$ счетно категорична, допускает элиминацию кванторов, разрешима и множество полных формул у нее вычислимо равномерно по $\mathrm{m}$.

ДокАзАтельство. Доказательство счетной категоричности следует из простой проверки изоморфизма любых двух счетных моделей этой теории челночным методом построения изоморфизма. Нужно заметить, что любая конечная модель теории $T_{0}^{m}$ может быть расширена до изоморфной $m$-допустимой и выполнено свойство амальгамируемости для конечных моделей теории $T_{0}^{m}$.

Из счетной категоричности и бесконечности ее моделей следует полнота и разрешимость этой теории. Отсюда и из конечности сигнатуры и элиминации кванторов сразу получаем вычислимость множества ее полных формул.

Определим теперь теорию $\widehat{T}_{Y}^{*}=\bigcup T(m, Y)$.

Рассмотрим теорию Палютина $T_{\mathbb{N}}$ для множества всех натуральных чисел, и пусть $g: \mathbb{N} \underset{\text { на }}{\stackrel{1-1}{\longrightarrow}} \mathbb{N}$ функция, по которой строится эквивалентность $n \stackrel{k}{\sim} m$ в этом случае, т. е. $\tilde{g}(k, n)=\tilde{g}(k, m)$.

Рассмотрим обратную к $g$, вычислимую функцию $h$. Определим $H(n)=$ $\max \{h(n), n\}+1$.

Пусть $\mathfrak{M}_{\mathbb{N}}-$ модель теории $T_{\mathbb{N}}$.

Пусть $M$ - основное множество модели $\mathfrak{M}_{\mathbb{N}}$. Определим модель $\mathfrak{M}_{\mathbb{N}}^{Y}$, взяв основное множество $M$ модели $\mathfrak{M}_{\mathbb{N}}$ в качестве основного множества.

Для любых $k \in \mathbb{N}$ и $m \in \mathbb{N}$ определим предикат $\left(P_{m}^{k+1}\right)_{\mathfrak{M}_{\mathbb{N}}^{Y}}$ для сигнатурного символа $\left(P_{m}^{k+1}\right)$ равным $\left(P_{0}^{k+1}\right)_{\mathfrak{M}_{\mathbb{N}}}$, если $m \stackrel{k}{\sim} \underset{\hat{f}}{ } 0$, и равным $\left(P_{H(k)}^{k+1}\right)_{\mathfrak{M}_{\mathbb{N}}}$, если не выполнено $m \stackrel{k}{\sim} \underset{\hat{f}}{0}$. Очевидно, что в построенной модели выполнены все аксиомы теории $T_{Y}$.

В [5], [6], следуя М. Г. Перетятькину [25], были определены понятие 2-ветвящегося дерева на множестве натуральных чисел и следующие функции и отношения на нем:

$$
\begin{gathered}
R(n) \leftrightharpoons 2 n+2, \quad L(n) \leftrightharpoons 2 n+1 ; \\
p(n) \leftrightharpoons \begin{cases}0, & \text { если } n=0, \\
{[(n-1) / 2]} & \text { в противном случае, }\end{cases}
\end{gathered}
$$


где $[x]$ - целая часть числа $x$;

$$
\begin{aligned}
& S(n) \leftrightharpoons \begin{cases}n-1, & \text { если } n>0 \text { и четно, } \\
n+1, & \text { если } n>0 \text { и нечетно, } \\
0 & \text { в противном случае; }\end{cases} \\
& h(n) \leftrightharpoons \begin{cases}0, & \text { если } n=0, \\
h(p(n))+1 & \text { в противном случае; }\end{cases} \\
& E_{p} \leftrightharpoons\{x \mid h(x)=p\} ;
\end{aligned}
$$

Отношение $x \preccurlyeq y$ определяется формулой

$$
\prod_{n=0}^{h(x)}|H(x, n)-y|=0
$$

и определяет на $\mathbb{N}$ порядок следующего типа:

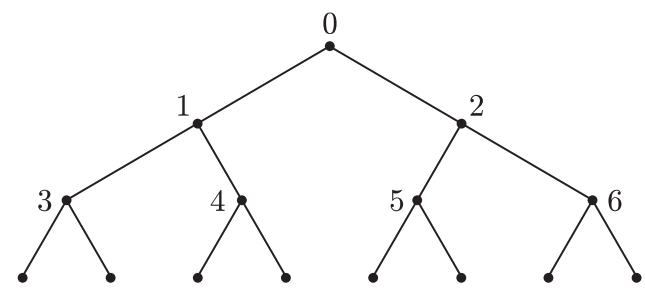

Рис. 1

Элемент $R(n)$ задает правый элемент под элементом $n$, а $L(n)$ - левый элемент под $n$, значение $p(n)$ задает для ненулевых элементов - ближайший элемент над $n$. Для пары элементов, лежащих под одним элементом, функция $S$ находит второй элемент пары, а функция $h(n)$ определяет число элементов над данным $n$.

Рассмотрим плотный линейный порядок без концов $(A, \leqslant)$ и определим на нем собственные начальные сегменты $\overline{\mathscr{D}}_{0}, \overline{\mathscr{D}}_{1}, \ldots, \overline{\mathscr{D}}_{n}, \ldots$ такие, что $\mathscr{D}_{0} \neq \varnothing$, $\overline{\mathscr{D}}_{i} \subset \overline{\mathscr{D}}_{i+1}, \bigcup \overline{\mathscr{D}}_{i}=A$ и для множества $\overline{\mathscr{D}}_{i}$ в $(A, \leqslant)$ нет точной верхней грани.

Построим теперь модель $\mathfrak{M}^{Y}$ теории $\widehat{T_{Y}^{*}}$. Определим интерпретацию предикатного символа $A$ равной множеству $A$, а интерпретацию $B$ равной множеству $M$, отношение $\leqslant$ М интерпретируем как порядок $\leqslant$ в $(A, \leqslant)$.

Полагаем предикатные символы $\mathscr{D}_{i}$ равными начальным сегментам $\overline{\mathscr{D}}_{i}$ в $A$.

Предикатный символ $P_{n}^{k+1}$ проинтерпретируем в $\mathfrak{M}^{Y}$ равным $\left(P_{0}^{k+1}\right)_{\mathfrak{M}_{\mathbb{N}}}$, если $n \stackrel{k}{\sim} \tilde{\hat{f}} 0$, и равным $\left(P_{H(k)}^{k+1}\right)_{\mathfrak{M}_{\mathbb{N}}}$, если не выполнено $n \stackrel{k}{\sim} \tilde{\hat{f}} 0$.

Определим по $h(n) \in \mathbb{N}$ плотный линейный порядок в $A \backslash \mathscr{D}_{h(n)}$ такой, что

1) $\eta_{0}$ - плотный линейный порядок в $A \backslash \mathscr{D}_{0}$ с плотным дополнением в $A \backslash \mathscr{D}_{0}$;

2 ) для всех $n \neq 0$ выполнены соотношения $\eta_{n} \cup \eta_{s(n)}=\eta_{p(n)}$ и $\eta_{n} \cap \eta_{s(n)}=\varnothing$, а $\eta_{n}$ и $\eta_{s(n)}$ - плотные подмножества в $\eta_{p(n)}$; 
3) $\left\{\eta_{n} \mid n \in \mathbb{N}\right\}$ - вычислимая последовательность рекурсивных подмножеств в $A$.

Определим для любого $n$ интерпретацию $\Delta_{n}$ и вспомогательные множества $\pi_{n}$ и $F_{n}$.

Полагаем $\Delta_{0}=\eta_{0}, F_{0}=\{1,2\}, \pi_{0}=\eta_{1}$, a

$$
\begin{aligned}
\Delta_{n+1} & =\bigcup_{k \in F_{n}} \eta_{L(k)}, \\
\pi_{n+1} & =\bigcup_{k \in F_{n}} \eta_{L L(k)}, \\
F_{n+1} & =\left\{L L(k), R L(k) \mid k \in F_{n}\right\} \quad \text { по рекурсии. }
\end{aligned}
$$

Так как $\left\{\eta_{n}, n \in \mathbb{N}\right\}$ - вычислимая последовательность рекурсивных множеств, то и $\left\{\Delta_{n}, n \in \mathbb{N}\right\}$ и $\left\{\pi_{n}, n \in \mathbb{N}\right\}$ - также вычислимые последовательности рекурсивных множеств, а последовательность $\left\{F_{n} \mid n \in \mathbb{N}\right\}$ конечных множеств сильно вычислима, т. е. мы еще можем вычислить и число элементов в $F_{n}$.

Полагаем

$$
\begin{gathered}
Q_{k}^{k+1}=\left\{\left(i, x_{1}, \ldots, x_{k+1}\right) \mid i \in \pi_{k} \text { и }\left(x_{1}, \ldots, x_{n}\right) \in\left(P_{H(n)}^{k+1}\right)_{\mathfrak{M}_{\mathbb{N}}}\right\} \\
\cup\left\{\left(i, x, \ldots, x_{k+1}\right) \mid i \in \Delta_{k} \backslash \pi_{k} \text { и }\left(x_{1}, \ldots, x_{k+1}\right) \notin\left(P_{H(n)}^{k+1}\right)_{\mathfrak{M}_{\mathbb{N}}},\right. \\
\text { а все элементы } \left.x_{1}, \ldots, x_{k+1} \text { из } B \text { попарно различны }\right\} .
\end{gathered}
$$

Нетрудно видеть, что в построенной модели $\mathfrak{M}_{\mathbb{N}}^{Y}$ выполнены все аксиомы теории $\widehat{T}_{Y}^{*}$. Таким образом, множество аксиом теории $\widehat{T}_{Y}^{*}$ непротиворечиво. А построенная модель $\mathfrak{M}^{Y}$ вычислима.

Из равномерности разрешимости теорий $T(m, Y), m \in \mathbb{N}$, и элиминации кванторов для них очевидным образом следует разрешимость и элиминация кванторов для теории $\widehat{T}_{Y}^{*}$.

Отсюда получаем, что построенная модель $\mathfrak{M}^{Y}$ теории $\widehat{T}_{Y}^{*}$ разрешима. Покажем, что она простая.

ЛЕмма 2. Любые две счетные модели теории $\widehat{T}_{Y}^{*}$, у которых $A=\bigcup_{n \geqslant 0}\left(\mathscr{D}_{n}\right)_{\mathfrak{M}}$,
оморфны.

ДокАЗАТЕЛЬство. Пусть $\mathfrak{M}_{1}$ и $\mathfrak{M}_{2}$ - две счетные модели теории $\widehat{T}_{Y}^{*}$ с условиями $M_{1}=\bigcup_{n \geqslant 0}\left(\mathscr{D}_{n}\right)_{\mathfrak{M}_{1}}$ и $M_{2}=\bigcup_{n \geqslant 0}\left(\mathscr{D}_{n}\right)_{\mathfrak{M}_{2}}$.

Рассмотрим пересчет $a_{0}, \ldots, a_{n}$ элементов $M_{1}$ и пересчет $b_{0}, \ldots, b_{n}, \ldots$ элементов $M_{2}$.

Заметим, что в этих моделях выполнены следующие свойства.

1. Если $\mathfrak{N}_{1}^{0}, \mathfrak{N}_{2}^{0}-m$-допустимые модели теории $T_{0}^{m}, \mathfrak{N}_{1}^{0} \subseteq \mathfrak{N}_{2}^{0}$, в $\mathfrak{M}_{1}$ и $\mathfrak{M}_{2}$ есть подмодели $\mathfrak{M}_{1}^{1} \subseteq \mathfrak{M}_{1}$ и $\mathfrak{M}_{2}^{1} \subseteq \mathfrak{M}_{2}$ и $\varphi-$ изоморфизм модели $\mathfrak{M}_{1}^{1}$ на $\mathfrak{M}_{2}^{1}$ и $\mathfrak{N}_{1}^{0} \cong \mathfrak{M}_{1}^{1}, \mathfrak{N}_{1}^{0} \cong \mathfrak{M}_{2}^{1}$, а $\mathfrak{M}_{1}^{2} \cong \mathfrak{N}_{2}^{0}$, причем все элементы из $A$ моделей $\mathfrak{M}_{1}^{1}$ и $\mathfrak{M}_{2}^{1}$ удовлетворяют предикату $\mathscr{D}_{m}$, а предикаты с индексами, большими $m$, в них ложны, кроме предикатов $\mathscr{D}_{k}$ при $k>m$, которые истинны на всех элементах, удовлетворяющих предикату $A$, то найдется подмодель $\mathfrak{M}_{2}^{2}$ в модели $\mathfrak{M}_{2}$ такая, 
что $\mathfrak{N}_{2}^{0} \cong \mathfrak{M}_{2}^{2}$ и существует продолжение $\psi^{1}$ изоморфизма $\varphi$ до изоморфизма модели $\mathfrak{M}_{1}^{2}$ на $\mathfrak{M}_{2}^{2}$.

2. Для любой конечной подмодели $\mathfrak{M}$ модели $\mathfrak{M}_{i}, i \in\{0,1\}$, существуют число $m \in \mathbb{N}$ и конечное расширение $\mathfrak{M}^{\prime}$ модели $\mathfrak{M}, \mathfrak{M}^{\prime} \supseteq \mathfrak{M}_{\text {и }} \mathfrak{M}^{\prime} \subseteq \mathfrak{M}_{i}$, такие, что $\mathfrak{M}^{\prime}$ является $m$-допустимой моделью, и на ее элементах все предикаты с индексами, большими $m$, ложны.

Из этих двух свойств челночным методом [31] очевидным образом строится требуемый изоморфизм.

Лемма 3. Для любой счетной модели $\mathfrak{M}$ теории $\widehat{T}_{Y}^{*}$ подмодель $\mathfrak{M}^{\prime}$ с основным множеством $\left\{a \mid\right.$ найдется $n$ такое, что $\left.\mathfrak{M}=\mathscr{D}_{n}(a)\right\} \cup B(\mathfrak{M})$ будет моделью теории $T_{Y}^{*}$.

Доказательство получается очевидным образом из элиминации кванторов простой проверкой всех аксиом.

Из лемм 1 и 2 заключаем, что построенная модель $\mathfrak{M}_{Y}$ проста и сильно конструктивизируема.

Заметим, что в построенной модели выполнены следующие свойства.

Свойство 1. Если $a_{1}, \ldots, a_{n}$ лежат в $\mathscr{D}_{m}\left(\mathfrak{M}_{Y}\right)$, а наборы $\left(b_{1}, \ldots, b_{k+1}\right)$, $\left(c_{1}, \ldots, c_{k+1}\right)$ удовлетворяют формуле $P_{0}^{k+1}$, где $k \notin Y, u$ в обеднении $\mathfrak{M}_{Y}$ до сигнатуры $\Sigma_{m}^{Y}$ они удовлетворяют одной и той же полной формуле

$$
\varphi\left(a_{1}, \ldots, a_{n}, x_{1}, \ldots, x_{k+1}\right)
$$

теории $\operatorname{Th}\left(\mathfrak{M}_{Y} \uparrow \Sigma_{m_{1}}^{Y}, a_{1}, \ldots, a_{n}\right)$, то существует автоморфизм $\varphi$ модели $\mathfrak{M}_{Y}$ такой, что $\varphi\left(a_{i}\right)=a_{i}$ для $i \leqslant n, a \varphi\left(b_{i}\right)=c_{i}$ для $1 \leqslant i \leqslant k+1$.

Свойство 2. Если $a_{1}, \ldots, a_{n}$ лежат в $\mathscr{D}_{m}\left(\mathfrak{M}_{x_{1}}\right)$, а наборь $\left(b_{1}, \ldots, b_{k+1}\right)$, $\left(c_{1}, \ldots, c_{k+1}\right)$ удовлетворяют в обеднении модели $\mathfrak{M}_{Y}$ до сигнатурь $\Sigma_{m}^{Y}$ одной и той же полной формуле теории $\operatorname{Th}\left(\mathfrak{M}_{Y} \uparrow \Sigma_{m}^{Y}, a_{1}, \ldots, a_{n}\right)$ u при $k>m$ выполнено $R^{*}\left(b_{1}, \ldots, b_{k+1}, c_{1}, \ldots, c_{k+1}\right)$, то существует автоморфизм $\varphi$ модели $\mathfrak{M}_{Y}$ такой, что $\varphi\left(a_{i}\right)=a_{i}$ для $1 \leqslant i \leqslant n, a \varphi\left(b_{i}\right)=c_{i}$ для $1<i \leqslant k+1$. $\underset{i=1}{k} \mathscr{D}_{m}\left(a_{i}\right)$, формула

Свойство 3. Для любых элементов $a_{1}, \ldots, a_{n}$ из $\mathfrak{M}_{Y} \operatorname{ma\kappa ux,~что~} \mathfrak{M}_{Y} \models$

$$
P_{0}^{k+1}\left(x_{1}, \ldots, x_{k+1}\right) \& \underset{i=0}{\&}\left(\underset{\bar{x} \subseteq\left\{x_{1}, \ldots, x_{k+1}\right\}}{\&} P_{0}^{i}(\bar{x})\right)
$$

является полной формулой теории $\operatorname{Th}\left(\mathfrak{M}_{Y}, a_{1}, \ldots, a_{n}\right)$ тогда и только тогда, когда $k \notin Y$. В силу этого свойства по критерию Нуртазина [6] получаем, что модель $\mathfrak{M}_{Y}$ не автоустойчива относителъно силъных конструктивизаций.

Определим теперь новую модель $\mathfrak{M}^{*}$ и константу $а$ в ней такую, что модель $\left(\mathfrak{M}^{*}, a\right)$ простая, но в ней множество полных формул вычислимо и, следовательно, она будет автоустойчивой относительно сильных конструктивизаций, а отсюда следует, что она будет автоустойчивой в силу элиминации кванторов.

Рассмотрим счетный плотный линейный рекурсивный порядок $(\mathscr{D}, \leqslant)$ без концов. 
Пусть $d_{0}, d_{1}, \ldots, d_{n}, \ldots$ - вычислимое перечисление всех его элементов. Определим вычислимое семейство его плотных рекурсивных подмножеств $\tilde{\eta}_{n}$, $n \in \mathbb{N}$, такое, что

1) $\tilde{\eta}_{0}$ и $\mathscr{D} \backslash \tilde{\eta}_{0}-$ плотные подмножества в $(\mathscr{D}, \leqslant), d_{0} \notin \tilde{\eta}_{0}$;

2 для любого $n$ выполнено $\tilde{\eta}_{L_{(n)}} \cup \tilde{\eta}_{R_{(n)}}=\tilde{\eta}_{n}, \tilde{\eta}_{L_{(n)}} \cap \tilde{\eta}_{R_{(n)}}=\varnothing$ и $d_{R_{(n)}} \notin \tilde{\eta}_{L_{(n)}}$;

3) полагаем $\widetilde{\Delta}_{0}=\tilde{\eta}_{0}, \tilde{\pi}_{0}=\tilde{\eta}_{1}$, а для всех $n$ из $\mathbb{N}$ полагаем

$$
\begin{aligned}
\widetilde{\Delta}_{n+1} & =\bigcup_{k \in F_{n}} \tilde{\eta}_{L_{(k)}}, \\
\tilde{\pi}_{n} & =\bigcup_{k \in F_{n}} \tilde{\eta}_{L L_{(k)}},
\end{aligned}
$$

где множества $F_{n}$ определены ранее.

Рассмотрим новый элемент $\alpha$, не лежащий ни в $\mathfrak{M}_{Y}$, ни в $\mathscr{D}$.

Расширим основное множество модели $\mathfrak{M}_{Y}$ с элементами из $\mathscr{D}$ и элементом $\alpha$ до множеств $M^{*}$. Это будет основное множество нашей модели $\mathfrak{M}^{*}$. Определим теперь на $M^{*}$ все основные предикаты сигнатуры модели $\mathfrak{M}_{Y}$.

Будем через $(S)_{\mathfrak{M}_{Y}}$ обозначать интерпретацию предикатного символа в $\mathfrak{M}_{Y}$, а через $(S)^{*}$ - интерпретацию этого символа в модели $\mathfrak{M}^{*}$.

Определим другие отношения на основном множестве, положив

$$
\begin{gathered}
(A)^{*} \leftrightharpoons(A)_{\mathfrak{M}_{Y}} \cup\{\alpha\} \cup \mathscr{D}, \quad(B)^{*} \leftrightharpoons(B)_{\mathfrak{M}_{Y}}, \quad\left(\mathscr{D}_{n}\right)^{*} \leftrightharpoons\left(\mathscr{D}_{n}\right)_{\mathfrak{M}_{Y}}, \\
(\leqslant)^{*} \leftrightharpoons(\leqslant)_{\mathfrak{M}_{Y}} \cup\left\{(x, \alpha) \mid x \in(A)_{\mathfrak{M}_{Y}}\right\} \cup\{(\alpha, y) \mid y \in \mathscr{D}\} \cup\{(\alpha, \alpha)\} \\
\left(P_{n}^{k+1}\right)_{\mathfrak{M}^{*}}^{*} \leftrightharpoons\left(P_{n}^{k+1}\right)_{\mathfrak{M}_{Y}}, \quad\left(\Delta_{n}\right)^{*}=\left(\Delta_{n}\right)_{\mathfrak{M}_{Y}} \cup\{\alpha\} \cup \widetilde{\Delta}_{n}, \\
\left(R_{n}\right)^{*}=\left(R_{n}\right)_{\mathfrak{M}_{Y}} \cup\left\{(x, y) \mid x, y \in \tilde{\pi}_{n} \cup \pi_{n}\right\} \\
\cup\left\{(x, y) \mid x, y \in\left(\Delta_{n} \cup \widetilde{\Delta}_{n}\right) \backslash\left(\tilde{\pi}_{n} \cup \pi_{n}\right)\right\} \cup\left\{(\alpha, x) \mid x \in \pi_{n} \cup \tilde{\pi}_{n}\right\}, \\
\left(R_{n}^{*}\right)^{*} \leftrightharpoons\left(R_{n}^{*}\right)_{\mathfrak{M}_{Y}}, \\
\left(Q_{k}^{k+1}\right)^{*} \leftrightharpoons\left(Q_{k}^{k+1}\right)_{\mathfrak{M}_{Y}} \cup\left\{(i, \bar{x}) \mid i \in \tilde{\pi}_{n} \text { и } x \in P_{H(k)}^{k}\right\} \\
\cup\left\{(i, \bar{x}) \mid i \in \widetilde{\Delta}_{n} \backslash \tilde{\pi}_{n} \text { и } \bar{x} \in\left(P_{0}^{k}\right)_{\mathfrak{M}_{Y}} \backslash P_{H(k)}^{k}\right\} .
\end{gathered}
$$

Нетрудно проверить, что все аксиомы теории $\widehat{T}_{Y}^{*}$ выполняются в модели $\mathfrak{M}^{*}$. Модель вычислима по построению, а из элиминации кванторов следует ее разрешимость и, следовательно, сильная конструктивизируемость.

ЛЕмма 4. Модель $\left(\mathfrak{M}^{*}, \alpha\right)$ простая.

ДокАЗАТЕЛьство. Для доказательства простоты покажем, что любой набор элементов из $\mathfrak{M}^{*}$ реализует главный тип.

Разобьем набор элементов из $\left(a_{1}, \ldots, a_{n}\right)$ на две части, где $a_{1}, \ldots, a_{r}$ - те элементы, которые удовлетворяют отношению $A$, и $\left(a_{r+1}, \ldots, a_{n}\right)$ - все остальные. Без ограничения общности можем предположить, что в этом наборе нет элемента $\alpha$, а также что все элементы попарно различны.

Пусть $a_{1}<\cdots<a_{s}<\alpha<a_{s+1}<\cdots<a_{r}$. Тогда найдутся $\mathscr{D}_{n_{i}}$ такие, что выполнено $\mathscr{D}_{n_{i}}\left(a_{i}\right)$ и $\neg \mathscr{D}_{n_{i+1}}\left(a_{i}\right)$. Пусть $m=\max \left\{n_{i} \mid i \leqslant r\right\}$. По построению найдутся наименьшие $m_{s+1}, \ldots, m_{k}$ такие, что выполнено $\neg \Delta_{m_{i}}\left(a_{i}\right)$ для $i \geqslant$ $s+1$. 
Рассмотрим теперь формулу $\psi_{\bar{a}}\left(\alpha, x_{1}, \ldots, x_{n}\right)$, взяв конъюнкцию следующих формул:

1) $\mathscr{D}_{n_{i}}\left(x_{i}\right) \& \neg \mathscr{D}_{n_{i+1}}\left(x_{i}\right)$ для $i \leqslant s$;

2) $x_{i}<\alpha$ для $i \leqslant s$;

3) $\alpha<x_{i}$ для $n \geqslant i \geqslant s+1$;

4) $R_{k}\left(\alpha, x_{i}\right)$ для $k \leqslant m$, если $\mathfrak{M}^{*}=R_{k}\left(\alpha, a_{i}\right)$ и $i \leqslant r$;

5) $\neg R_{k}\left(\alpha, x_{i}\right)$ для $k \leqslant m$, если $\mathfrak{M}^{*}=\neg R_{k}\left(\alpha, a_{i}\right)$ и $i \leqslant r$;

6) $Q_{k}^{k+1}\left(\alpha, x_{i_{1}}, \ldots, x_{i_{k+1}}\right)$, где $r \geqslant i_{j} \geqslant s+1$, а $k \leqslant n+1$ и

$$
\mathfrak{M}^{*} \models Q_{k}^{k+1}\left(\alpha, a_{i_{1}}, \ldots, a_{i_{k+1}}\right) ;
$$

7) $\neg Q_{k}^{k+1}\left(\alpha, x_{i_{1}}, \ldots, x_{i_{k+1}}\right)$, где $i_{j} \geqslant r+1$, а $k \leqslant n+1$ и

$$
\mathfrak{M}^{*} \models \neg Q_{k}^{k+1}\left(\alpha, a_{i_{1}}, \ldots, a_{i_{k+1}}\right) ;
$$

8) $P_{0}^{k+1}\left(x_{i}, \ldots, x_{i_{k+1}}\right)$, где $i_{j} \geqslant r+1$, а $k \leqslant n+1$ и

$$
\mathfrak{M}^{*} \models P_{0}^{k+1}\left(a_{i_{1}}, \ldots, a_{i_{k+1}}\right) ;
$$

9) $\neg P_{0}^{k+1}\left(x_{i}, \ldots, x_{i_{k+1}}\right)$, где $i_{j} \geqslant s+1$, а $k \leqslant n+1$ и

$$
\mathfrak{M}^{*} \models \neg P_{0}\left(a_{i_{1}}, \ldots, a_{i_{k+1}}\right) .
$$

Нетрудно челночным методом показать, что для любых наборов $\left(c_{1}, \ldots, c_{n}\right)$ и $\left(b_{1}, \ldots, b_{n}\right)$, которые удовлетворяют формуле $\Psi_{\bar{a}}\left(\alpha, x_{1}, \ldots, x_{n}\right)$, существует автоморфизм $\varphi$ модели $\mathfrak{M}_{Y}$, оставляющий $\alpha$ на месте, такой, что $\varphi\left(c_{i}\right)=d_{i}$. Отсюда следует полнота этой формулы и, следовательно, тип - главный. Лемма доказана.

Заметим, что построение формулы $\Psi_{\bar{a}}$ по набору $\bar{a}$ вычислимо и, следовательно, мы построили искомое семейство вычислимых полных формул, но в таком случае, по критерию Нуртазина (теорема 1), модель $\mathfrak{M}^{*}$ автоустойчива относительно сильных конструктивизаций. Существование континуума различных типов в нашей теории очевидно в силу аксиомы Q5.

Теорема 7 доказана.

Докажем теперь, что теория с неавтоустойчивой относительно сильных конструктивизаций простой моделью, но с некоторой автоустойчивой относительно сильных конструктивизаций моделью будет иметь сложное теоретико-модельное устройство.

ТЕОРема 8. Если Т имеет простую сильно конструктивизируемую неавтоустойчивую относительно сильных конструктивизаций модель, а некоторая модель теории Т автоустойчива относительно силъных конструктивизаций, то теория $T$ не $\mathbb{N}$-стабильна.

ДоКАЗАТЕЛЬСТВО. Пусть $(\mathfrak{N}, \nu)$ - сильно конструктивная простая модель теории $T, \mathfrak{N} \preccurlyeq \mathfrak{M}$ и модель $\mathfrak{M}$ имеет сильную конструктивизацию и автоустойчива относительно сильных конструктивизаций. 
По теореме А. Т. Нуртазина [6] существует конечный набор $\bar{a}$ элементов из $\mathfrak{M}$ такой, что модель $(\mathfrak{M}, \bar{a})$ простая и семейство полных формул вычислимо, а в $\mathfrak{N}$ при любом обогащении конечным числом констант для элементов $b_{1}, \ldots, b_{k}$ в обогащении $\left(\mathfrak{N}, b_{1}, \ldots, b_{k}\right)$ семейство полных формул не вычислимо.

Рассмотрим $p\left(x_{1}, \ldots, x_{n}\right)$ - тип элементов $\bar{a}=\left(a_{1}, \ldots, a_{n}\right)$ в модели $\mathfrak{M}$.

Рассмотрим $\varphi_{0}\left(\bar{y}_{0}\right)$ - полную формулу в теории $\operatorname{Th}(\mathfrak{N})$ такую, что $\varphi_{0}\left(\bar{y}_{0}\right)$ не является полной в модели $\left(\mathfrak{M}, a_{1}, \ldots, a_{n}\right)$. Такая формула существует, так как в противном случае в модели $\mathfrak{N}$ множество полных формул было бы вычислимо, поскольку в $\left(\mathfrak{M}, a_{1}, \ldots, a_{n}\right)$ множество полных формул вычислимо.

Модель $\left(\mathfrak{M}, a_{1}, \ldots, a_{n}\right)$ простая. Следовательно, существует полная формула $\xi_{0}\left(a_{1}, \ldots, a_{n}, y_{1}, \ldots, y_{k}\right)$ такая, что

$$
\begin{aligned}
\operatorname{Th}\left(\mathfrak{M}, a_{1}, \ldots, a_{n}\right) \models & (\forall \bar{y})\left(\xi_{0}(\bar{a}, \bar{y}) \rightarrow \varphi_{0}(\bar{y})\right) \\
& \&(\exists \bar{y})\left(\varphi_{0}(\bar{y}) \& \neg \xi_{0}(\bar{a}, \bar{y}) \&(\exists y) \xi_{0}(\bar{a}, \bar{y})\right) .
\end{aligned}
$$

Рассмотрим набор $\bar{b}_{0}$ из подмодели $\mathfrak{N}$ такой, что выполнено $\mathfrak{N}=\varphi_{0}\left(b_{0}\right)$, но $\mathfrak{M} \models \neg \xi_{0}\left(\bar{a}_{0}, \bar{b}_{0}\right)$, где $\bar{a}_{0}$ обозначает набор элементов $a_{1}, \ldots, a_{n}$.

На первом шаге рассмотрим множества

$$
\begin{gathered}
q_{0}\left(\bar{b}_{0}\right)=\left\{\varphi_{0}\left(\bar{b}_{0}\right)\right\}, \\
q^{0}\left(\bar{x}, \bar{x}^{\prime}, \bar{b}_{0}\right)=p(\bar{x}) \cup p\left(\bar{x}^{\prime}\right) \cup\left\{\bar{x} \neq \bar{x}^{\prime}\right\} \cup\left\{\xi_{0}\left(\bar{x}, \bar{b}_{0}\right)\right\} \cup\left\{\neg \xi_{0}\left(\bar{x}^{\prime}, \bar{b}_{0}\right)\right\} \cup\left\{\varphi_{0}\left(\bar{b}_{0}\right)\right\}, \\
q^{1}\left(\bar{x}, \bar{x}^{\prime}, \bar{b}_{0}\right)=p(\bar{x}) \cup p\left(\bar{x}^{\prime}\right) \cup\left\{x \neq x^{\prime}\right\} \cup\left\{\neg \xi_{0}\left(\bar{x}, \bar{b}_{0}\right)\right\} \cup\left\{\xi_{0}\left(\bar{x}^{\prime}, \bar{b}_{0}\right)\right\} \cup\left\{\varphi_{0}\left(\bar{b}_{0}\right)\right\} .
\end{gathered}
$$

Заметим, что $q_{0} \subseteq q^{0}$ и $q_{0} \subseteq q^{1}$, а множество $q^{0} \cup q^{1}$ не совместно. Покажем, что множества $q^{0}$ и $q^{1}$ совместны с $\operatorname{Th}\left(\mathfrak{N}, \bar{b}_{0}\right)$.

Рассмотрим произвольную формулу $\Delta(\bar{x})$ из $p(\bar{x})$. Существует формула $\Delta^{\prime}$ из $p(\bar{x})$ такая, что

$$
\begin{aligned}
\operatorname{Th}(\mathfrak{N}) \models(\forall \bar{x})\left(\Delta^{\prime}(\bar{x}) \rightarrow \Delta(\bar{x})\right) & \&(\forall \bar{x})(\forall \bar{y})\left(\Delta^{\prime}(\bar{x}) \& \xi_{0}(\bar{x}, \bar{y}) \rightarrow \varphi_{0}(\overline{\bar{y}})\right) \\
& \&(\exists \bar{x} \bar{y})\left(\Delta^{\prime}(\bar{x} \bar{y}) \& \xi_{0}(\bar{x}, y) \& \varphi_{0}(y)\right) .
\end{aligned}
$$

Так как $\varphi_{0}(\bar{y})$ - полная формула в $\operatorname{Th}(\mathfrak{N})$, то в $\operatorname{Th}(\mathfrak{N})$ выполнена формула

$$
(\forall \bar{y})\left(\varphi_{0}(\bar{y}) \rightarrow(\exists \bar{x})\left(\Delta^{\prime}(\bar{x}) \& \xi_{0}(\bar{x}, \bar{y}) \& \varphi_{0}(\bar{y})\right)\right) .
$$

Тогда найдется набор $\bar{c}$ такой, что

$$
\mathfrak{N} \models \Delta^{\prime}(\bar{c}) \& \xi_{0}\left(\bar{c}, \bar{b}_{0}\right) \& \varphi_{0}\left(\bar{b}_{0}\right) .
$$

Для набора $\bar{a}_{0}$ в $\mathfrak{M}$ выполнены формулы

$$
\Delta^{\prime}\left(a_{0}\right) \& \Delta^{\prime}(\bar{c}) \& \xi_{0}\left(\bar{c}, \bar{b}_{0}\right) \& \neg \xi_{0}\left(\bar{a}_{0}, \bar{b}_{0}\right) \& \bar{c} \neq \bar{a}_{0} \& \varphi_{0}\left(\bar{b}_{0}\right)
$$

и

$$
\Delta\left(\bar{a}_{0}\right) \& \Delta(\bar{c}) \& \xi_{0}\left(\bar{c}, \bar{b}_{0}\right) \& \neg \xi_{0}\left(\bar{a}_{0}, \bar{b}_{0}\right) \& \varphi\left(\bar{b}_{0}\right) .
$$

Так как $p(\bar{x})$ - тип, то для любого его конечного подмножества $p^{\prime}(\bar{x})$ найдется формула $\Delta(\bar{x})$ из $p(\bar{x})$ такая, что

$$
\mathfrak{N} \models(\forall \bar{x})\left(\Delta(\bar{x}) \rightarrow \& p^{\prime}(\bar{x})\right) .
$$


Отсюда по теореме А.И. Мальцева о компактности заключаем, что $q^{0}$ и $q^{1}$ совместны с $\operatorname{Th}\left(\mathfrak{N}, \bar{b}_{0}\right)$.

Пусть определены наборы элементов $\bar{b}_{0}, \bar{b}_{1}, \bar{b}_{2}, \ldots, \bar{b}_{n}$ из модели $\mathfrak{N}$ и полные формулы $\varphi_{0}, \varphi_{1}, \varphi_{2}, \ldots, \varphi_{n}$ такие, что в $\mathfrak{N}$ выполнены $\varphi_{0}$ на $\bar{b}_{0}, \varphi_{1}$ на $\left(\bar{b}_{0}, \bar{b}_{1}\right)$, $\ldots, \varphi_{n}$ на $\left(\bar{b}_{0}, \bar{b}_{1}, \ldots, \bar{b}_{n}\right)$, и существуют полные формулы

$$
\xi_{0}\left(\bar{a}_{0}, \bar{y}_{0}\right), \xi_{1}\left(\bar{a}_{0}, \bar{b}_{0}, \bar{y}_{1}\right), \ldots, \xi_{n}\left(\bar{a}, \bar{b}_{0}, \ldots, \bar{y}_{n+1}\right)
$$

в модели $\mathfrak{M}$ с выделенными константными символами такие, что выполнены условия

$$
\begin{aligned}
\mathfrak{M}= & \left(\forall \bar{y}_{i}\right)\left(\xi_{i}\left(\bar{a}, \bar{b}_{0}, \ldots, \bar{b}_{i-1}, \bar{y}_{i}\right) \rightarrow \varphi_{i}\left(\bar{b}_{0}, \ldots, \bar{b}_{i-1}, \bar{y}_{i}\right)\right) \\
& \&\left(\exists \bar{y}_{i}\right)\left(\neg \xi_{i}\left(\bar{a}, \bar{b}_{0}, \ldots, b_{i-1}, \bar{y}_{i}\right) \& \varphi_{i}\left(\bar{b}_{0}, \ldots, \bar{b}_{i-1}, \bar{y}_{i}\right)\right) \\
& \&\left(\exists \bar{y}_{i}\right)\left(\xi_{i}\left(\bar{a}, \bar{b}_{0}, \ldots, \bar{b}_{i-1}, \bar{y}_{i}\right) \& \varphi_{i}\left(\bar{b}_{0}, \ldots, \bar{b}_{i-1}, \bar{y}_{i}\right)\right)
\end{aligned}
$$

И

$$
\mathfrak{M} \models \neg \xi_{k}\left(\bar{a}, \bar{b}_{0}, \ldots, \bar{b}_{i-1}, \bar{b}_{i}\right),
$$

а множества

$$
\begin{aligned}
q^{\varepsilon_{0}, \ldots, \varepsilon_{n}}\left(\bar{x}, \bar{x}^{\prime}, \bar{b}_{0}, \bar{b}_{n}\right) & =p(\bar{x}) \cup p\left(\bar{x}^{\prime}\right) \cup\left\{\bar{x} \neq \bar{x}^{\prime}\right\} \\
& \cup\left\{\xi_{i}^{\varepsilon_{i}}\left(\bar{x}, \bar{b}_{0}, \ldots, \bar{b}_{i}\right), \xi_{i}^{1-\varepsilon_{i}}\left(\bar{x}^{\prime}, \bar{b}_{0}, \ldots, \bar{b}_{i}\right), \varphi_{i}\left(\bar{b}_{0}, \ldots, \bar{b}_{i}\right) \mid i \leqslant n\right\}
\end{aligned}
$$

для любого набора $\left(\varepsilon_{0}, \ldots, \varepsilon_{i}\right) \in\{0,1\}^{i+1}$ совместны с $\operatorname{Th}\left(\mathfrak{N}, \bar{b}_{0}, \ldots, \bar{b}_{n}\right)$.

Из полноты выбранных формул заключаем, что для любых формул $\xi\left(\bar{x}_{0}, \bar{b}_{0}\right.$, $\left.\ldots, \bar{b}_{n}\right)$ и $\eta\left(\bar{x}, \bar{b}_{0}, \ldots, \bar{b}_{n}\right)$ таких, что $\xi\left(\bar{a}, \bar{b}_{0}, \ldots, \bar{b}_{n}\right)$ и $\eta\left(\bar{a}, b_{0}, \ldots, b_{n}\right)$ выполняются в модели $\mathfrak{M}$, множества

$$
p^{\varepsilon_{0}, \ldots, \varepsilon_{n}}\left(\bar{x}, \bar{x}^{\prime}, \bar{b}_{0}, \ldots, \bar{b}_{n}\right) \cup\left\{\xi\left(\bar{x}, \bar{b}_{0}, \ldots, \bar{b}_{n}\right), \eta\left(\bar{x}^{\prime}, \bar{b}_{0}, \ldots, \bar{b}_{n}\right)\right\}
$$

совместны с $\operatorname{Th}\left(\mathfrak{N}, \bar{b}_{0}, \ldots, \bar{b}_{n}\right)$.

Заметим, что для $\left(\varepsilon_{0}, \ldots, \varepsilon_{n}\right) \neq\left(\varepsilon_{0}^{\prime}, \ldots, \varepsilon_{n}^{\prime}\right)$ множества

$$
q_{0}^{\varepsilon_{0}, \ldots, \varepsilon_{n}}\left(\bar{x}, \bar{x}^{\prime}, \bar{b}_{0}, \ldots, \bar{b}_{n}\right) \cup q^{\varepsilon_{0}^{\prime}, \ldots, \varepsilon_{n}^{\prime}}\left(\bar{x}, \bar{x}^{\prime}, \bar{b}_{0}, \ldots, \bar{b}_{n}\right)
$$

не совместны с $\operatorname{Th}(\mathfrak{N})$.

Определим теперь искомые наборы и типы для $n+1$.

Рассмотрим обогащение $\mathfrak{N}$ найденными для $n$ элементами $\bar{b}_{0}, \ldots, \bar{b}_{n}$. Тогда существует полная формула $\varphi_{n+1}\left(\bar{y}_{0}, \bar{y}_{1}, \ldots, \bar{y}_{n}, \bar{y}_{n+1}\right)$ такая, что

$$
\mathfrak{N} \models\left(\exists \bar{y}_{n+1}\right) \varphi_{n+1}\left(\bar{b}_{0}, \ldots, \bar{b}_{n}, \bar{y}_{n+1}\right),
$$

но формула $\varphi_{n+1}\left(\bar{b}_{0}, \ldots, \bar{b}_{n}, \bar{y}_{n+1}\right)$ не является полной в обогащении $(\mathfrak{M}, \bar{a}$, $\left.\bar{b}_{0}, \ldots, \bar{b}_{n}\right)$. Рассмотрим полную формулу $\xi_{n+1}\left(\bar{a}, \bar{b}_{0}, \ldots, \bar{b}_{n}, \bar{y}_{n+1}\right)$ такую, что в $\left(\mathfrak{M}, \bar{a}, \bar{b}_{0}, \ldots, \bar{b}_{n}\right)$ выполнены формулы

$$
\begin{gathered}
\left(\forall \bar{y}_{n+1}\right)\left(\xi_{n+1}\left(\bar{a}, \bar{b}_{0}, \ldots, \bar{b}_{n}, \bar{y}_{n+1}\right) \rightarrow \varphi_{n+1}\left(\bar{b}_{0}, \ldots, \bar{b}_{n}, \bar{y}_{n+1}\right)\right), \\
\left(\exists \bar{y}_{n+1}\right)\left(\varphi_{n+1}\left(\bar{b}_{0}, \ldots, \bar{b}_{n}, \bar{y}_{n+1}\right) \& \neg \xi_{n+1}\left(\bar{a}, \bar{b}_{0}, \ldots, \bar{b}_{n}, \bar{y}_{n+1}\right)\right), \\
\left(\exists \bar{y}_{n+1}\right) \xi_{n+1}\left(\bar{a}, \bar{b}_{0}, \ldots, \bar{b}_{n}, \bar{y}_{n+1}\right) .
\end{gathered}
$$


В таком случае без ограничения общности можно считать, что найдется набор элементов $\bar{b}_{n+1}$ из подмодели $\mathfrak{N}$ такой, что $\mathfrak{N} \models \varphi_{n+1}\left(\bar{b}_{0}, \ldots, \bar{b}_{n}, \bar{b}_{n+1}\right)$, но $\mathfrak{M} \models \neg \xi_{n+1}\left(\bar{a}, \bar{b}_{0}, \ldots, \bar{b}_{n}, \bar{y}_{n+1}\right)$, поскольку $\mathfrak{N}$ - элементарная подмодель в $\mathfrak{M}$ и простая.

Определим теперь множества формул

$$
q^{\varepsilon_{0}, \ldots, \varepsilon_{n}, 0} \leftrightharpoons q^{\varepsilon_{0}, \ldots, \varepsilon_{n}} \cup\left\{\xi_{n+1}\left(\bar{x}, \bar{b}_{n+1}\right), \neg \xi_{n+1}\left(\bar{x}^{\prime}, \bar{b}_{n+1}\right), \varphi_{n+1}\left(\bar{b}_{0}, \ldots, \bar{b}_{n}, \bar{b}_{n+1}\right)\right\}
$$

и

$$
q^{\varepsilon_{0}, \ldots, \varepsilon_{n}, 1} \leftrightharpoons q^{\varepsilon_{0}, \ldots, \varepsilon_{n}} \cup\left\{\neg \xi_{n+1}\left(\bar{x}, \bar{b}_{n+1}\right), \xi_{n+1}\left(\bar{x}^{\prime}, \bar{b}_{n+1}\right), \varphi_{n+1}\left(\bar{b}_{0}, \ldots, \bar{b}_{n}, \bar{b}_{n+1}\right)\right\} .
$$

Как и на первом шаге, нужно заметить, что оба так определенных набора локально совместны. Так как определение этих множеств симметрично, то достаточно доказать локальную совместность одного из них, например $q^{\varepsilon_{0}, \ldots, \varepsilon_{n}, 0}$.

Для доказательства этого шага индукции рассмотрим конечное подмножество $q$ из $q^{\varepsilon_{0}, \ldots, \varepsilon_{n}}$. Из определения этих множеств следует, что множество $q$ может быть разбито на две части $q_{0}(\bar{x})$ и $q_{1}\left(\bar{x}^{\prime}\right), q=q_{0}(\bar{x}) \cup q_{1}(\bar{x})$, где $q_{0}(\bar{x})$ состоит из формул со свободными переменными из $\bar{x}$, а $q_{1}\left(\bar{x}^{\prime}\right)$ - из формул со свободными переменными из $\bar{x}^{\prime}$.

Нетрудно увидеть из построения этих множеств, что в модели $\left(\mathfrak{M}, \bar{b}_{0}, \ldots, \bar{b}_{n}\right)$ выполнены формулы из $q_{0}(\bar{x}) \cup\left\{\xi_{n+1}\left(\bar{x}, \bar{b}_{n+1}\right), \varphi_{n+1}\left(\bar{b}_{0}, \ldots, \bar{b}_{n}, \bar{b}_{n+1}\right)\right\}$ на набоpe $\bar{a}_{0}$, а из полноты формулы $\varphi_{n+1}\left(\bar{y}_{0}, \bar{y}_{1}, \ldots, \bar{y}_{n}, \bar{y}_{n+1}\right)$ следует, что в моделях $\left(\mathfrak{N}, \bar{b}_{0}, \ldots, \bar{b}_{n}\right)$ и $\left(\mathfrak{M}, \bar{b}_{0}, \ldots, \bar{b}_{n}\right)$ выполнена формула

$$
\left(\exists \bar{x}^{\prime}\right)\left(\& q_{1}\left(\bar{x}^{\prime}\right) \& \neg \xi_{n+1}\left(\bar{x}, \bar{b}_{n+1}\right) \& \varphi_{n+1}\left(\bar{b}_{0}, \ldots, \bar{b}_{n}, \bar{b}_{n+1}\right)\right) .
$$

Тогда множества формул

$$
q^{\varepsilon_{0}, \ldots, \varepsilon_{n}, 0} \leftrightharpoons q^{\varepsilon_{0}, \ldots, \varepsilon_{n}} \cup\left\{\xi_{n+1}\left(\bar{x}, \bar{b}_{n+1}\right), \neg \xi_{n+1}\left(\bar{x}^{\prime}, \bar{b}_{n+1}\right), \varphi_{n+1}\left(\bar{b}_{0}, \ldots, \bar{b}_{n}, \bar{b}_{n+1}\right)\right\}
$$

И

$$
q^{\varepsilon_{0}, \ldots, \varepsilon_{n}, 1} \leftrightharpoons q^{\varepsilon_{0}, \ldots, \varepsilon_{n}} \cup\left\{\neg \xi_{n+1}\left(\bar{x}, \bar{b}_{n+1}\right), \xi_{n+1}\left(\bar{x}^{\prime}, \bar{b}_{n+1}\right), \varphi_{n+1}\left(\bar{b}_{0}, \ldots, \bar{b}_{n}, \bar{b}_{n+1}\right)\right\}
$$

будут локально совместны и, следовательно, по теореме Мальцева о компактности, совместны с $\operatorname{Th}\left(\mathfrak{N}, \bar{b}_{0}, \ldots, \bar{b}_{n+1}\right)$.

Таким образом, мы можем рассмотреть произвольную цепь $L$ из 2-ветвящегося дерева $(N, \prec)$ и определить

$$
p_{L}^{\left(\bar{x}, \bar{x}^{\prime}\right)}=\bigcup_{\left(\varepsilon_{0}, \ldots, \varepsilon_{n}\right) \in L} p^{\varepsilon_{0}, \ldots, \varepsilon_{n}}\left(\bar{x}, \bar{x}^{\prime}, \bar{b}_{0}, \ldots, \bar{b}_{n}\right) .
$$

Тогда мы получаем над обогащением модели $\mathfrak{N}$ счетным множеством констант из $\bar{b}_{0}, \bar{b}_{1}, \ldots, \bar{b}_{n}, \ldots$ континуум различных типов. Отсюда следует, что теория $T$ не является $\mathbb{N}$-стабильной. Теорема 8 доказана.

СледСтвиЕ 1. Не существует несчетно категоричной разрешимой теории, у которой простая модель неавтоустойчива относительно сильных конструктивизаций, но некоторая другая автоустойчива относительно сильных конструктивизаций. 
СлЕДСтвиЕ 2. Если некоторая модель несчетно категоричной теории автоустойчива относительно сильных конструктивизаций, то и простая модель автоустойчива относительно сильных конструктивизаций.

Вопреки ожиданиям оказывается, что и в случае теорий со счетным числом счетных моделей справедлива следующая теорема. Однако вопрос о существовании эренфойхтовой теории, простая модель которой сильно конструктивизируема и неавтоустойчива относительно сильных конструктивизаций, но у которой существует простая модель в конечном обогащении константами, сильно конструктивизируемая и автоустойчивая относительно сильных конструктивизаций, остается открытым.

ТЕОрема 9. Существует полная разрешимая теория со счетным числом счетных моделей, простая модель которой сильно конструктивизируема и неавтоустойчива относительно сильных конструктивизаций, но у которой существует простая модель в конечном обогащении константами, сильно конструктивизируемая и автоустойчивая относительно сильных конструктивизаций.

ДокАзАТЕльство. Как в приведенном выше доказательстве теоремы Кудайбергенова [7], построим вначале серию примеров разрешимых счетно категоричных теорий с равномерно вычислимыми семействами полных формул. За счет встраивания конструкции плотного 2-ветвящегося дерева удается избавиться от континуума счетных моделей в строящейся теории.

Рассмотрим рекурсивно перечислимое множество $Y$ такое, что $0 \notin Y$, и строго вычислимую последовательность конечных множеств $\left\{Y^{t} \mid t \in \mathbb{N}\right\}$ такую, что $Y^{0}=\varnothing, Y^{t+1} \backslash Y^{t}$ содержит не более одного элемента и все элементы из $Y^{t}$ меньше $t$. Для каждого $Y^{t}$ определим функцию $g_{t}$, положив для каждого $y \in Y^{t}$ значение $g_{t}(y)$ равным наименьшему $z$ такому, что $y \in Y^{z}$.

Для множества $Y^{m} \subseteq\left[0, m\left[\right.\right.$ определим функцию $g_{m}: Y^{m} \rightarrow[0, m[$ такую, что для любых разных $x, y$ из $Y^{m}$ выполнено $g_{m}(x) \neq g_{m}(y)$, положив $g_{m}(x)$, $x \in Y^{m}$, равным наименьшему $s$ такому, что $x \in Y^{s}$.

Зададим сигнатуру теории $T^{m}\left(g_{m}, Y^{m}\right)$ для функции $g_{m}$ и множества $Y^{m}$, состоящую из одноместных предикатов $A, B, C$ для $0 \leqslant i \leqslant m$, бинарных отношений $\leqslant A, \leqslant_{A B}^{*}, \leqslant_{B}, \wedge_{B}$, предикатных символов $S_{k}^{i+2}$ местности $k+2$ для $0 \leqslant k \leqslant m, R_{n}^{k+1}$ для $0 \leqslant n \leqslant m, 0 \leqslant k \leqslant m$, одноместных предикатов $A_{0}, \ldots, A_{m}, B_{0}, \ldots, B_{m}$, а также бинарного предикатного символа $F$, бинарных предикатных символов $\Delta_{n}$, четырехместных предикатных символов $G_{n}$, двухместных предикатных символов $\bar{G}_{n}$, одноместных предикатных символов $E_{n}$ для $n \leqslant m$.

Зададим теперь аксиомы нашей теории $T^{m}\left(g_{m}, Y^{m}\right)$.

1. $A, B, C$ определяют разбиение основного множества на три бесконечные непересекающиеся части.

2. Отношение $\leqslant_{A}$ задает на $A$ плотный линейный порядок без наименьшего и наибольшего элементов.

3. Отношение $\leqslant_{B}$ задает на $B$ структуру плотного бинарного дерева [25] без наименьшего элемента, где $x \wedge_{B} y=x \Leftrightarrow x \leqslant_{B} y$. 
4. Отношение $F$ задает график отображения $B$ на $A$ такой, что если $x<_{B} y$, $x, y \in B$, то $F(x)<F(y)$, а в $F^{-1}(x)$ все элементы не сравнимы относительHо $\leqslant B$.

5. Для любых $x \in B$ и $z, y \in A$ верны импликации

1) $F(y)=x$ и $x<z \Rightarrow(\exists u)(F(u)=z) \& y<u$,

2) $F(y)=x$ и $z<x \Rightarrow(\exists u)(u<y \& F(u)=x)$.

6 . Отношение $\leqslant A B$ определено на $A \cup B$ так, что

если $x, y \in A$, то $x \leqslant_{A} y \Leftrightarrow x \leqslant_{A B} y$,

если $x, y \in B$, то $x \leqslant A B y \Leftrightarrow F(x) \leqslant F(y)$,

если $x \in A, y \in B$, то $x \leqslant_{A B} y \Leftrightarrow x \leqslant F(y)$ и $y \leqslant A B x \Leftrightarrow F(y) \leqslant x$.

7. Определено бинарное отношение $={ }_{B}$ на $B$ такое, что $x={ }_{B} y \Leftrightarrow F(x)=$ $F(y)$

8. $B_{n}(x) \Leftrightarrow F(x) \in A_{n}(x)$.

9. $A_{n}$ - начальный сегмент в $\left(A, \leqslant_{A}\right)$ и не существует точной верхней грани для $A_{n}$.

10. На элементах из $C$ относительно предикатов $R_{n}^{k+1}, D_{k}$ для $k, n$ из $\mathbb{N}$ определим ограничение на эту сигнатуру модифицированной теории Палютина $T_{m}\left(g_{m}, Y^{m}\right)$.

11.0. Для предикатного символа $\Delta_{0}$ определим аксиомы, что он определяет отношение эквивалентности из двух классов на множестве $B \backslash B_{0}$, причем для любого элемента $x$ из $B_{0}$ над ним в $B \backslash B_{0}$ есть элементы из обоих классов и для любых двух элементов $x, y$ из $B \backslash B_{0}$, если $x$ меньше $y$ в $B \backslash B_{0}$, то оба они лежат в одном классе эквивалентности $\Delta_{0}$. Для предикатного символа $E_{0}$ полагаем, что он эквивалентен $B \& \neg B_{0}$, а для предикатного символа $S_{0}$ определяем $S_{0}(x, \bar{z})$ так, что для каждого $x$ из $\Delta_{0}$-класса эквивалентных элементов $S_{0}(x, \bar{z})$ определяют одно и то же подмножество множества $R_{0}^{1}$, совпадающее с одним из классов $D_{0}$-эквивалентности, а для элементов из другого класса его дополнение в $R_{0}^{1}$. Причем для всех классов, для элементов которых $S_{0}(x, \bar{z})$ определено, определимых подмножеств только два и они либо совпадают, либо являются взаимодополняющими до $R_{0}^{1}$, а для всех $x$ не из $E_{0}$ предикат $S_{0}(x, \bar{z})$ ложен при любом $\bar{z}$.

11. $n(n<m)$. В этом наборе аксиом возможны два случая.

Случай 1. Если $Y^{n+1} \backslash Y^{n}=\varnothing$. В этом случае полагаем, что $E_{n+1}$ эквивалентен $E_{n} \& \neg B_{n+1}$. Для каждого класса $\Delta_{n}$-эквивалентных элементов, содержащего элементы из $E_{n}$, предикат $\Delta_{n+1}$ определяет на пересечении этого класса $\Delta_{n}$-эквивалентных элементов с $E_{n}$ точно два класса $\Delta_{n+1}$-эквивалентных элементов. Предикат для символа $S_{n+1}$ определяем так, что $S_{n+1}(x, \bar{z})$ выполнен для каждого $x$ из одного из этих $\Delta_{n+1}$-классов эквивалентных элементов, $S_{n+1}(x, \bar{z})$ определяют одно и то же подмножество множества $R_{n+1}^{n+2}$, совпадающее с одним из классов $D_{n+1}$-эквивалентности, а для элементов из другого класса - его дополнение в $R_{n+1}^{n+2}$. Причем для всех классов, для элементов которых $S_{n+1}(x, \bar{z})$ определено, определимых подмножеств только два и они либо совпадают, либо являются взаимодополняющими до $R_{n+1}^{n+2}$, а для всех $x$ не из $E_{n+1}$ предикат $S_{n+1}(x, \bar{z})$ ложен при любом $\bar{z}$. Кроме того, для любого элемента $x$ из $B_{n+1}$ над ним в $B \backslash B_{n+1}$ есть элементы из обоих классов и для 
любых двух элементов $x, y$ из $B \backslash B_{n+1}$, если $x$ меньше $y$ в $B \backslash B_{n+1}$, то оба они лежат в одном классе эквивалентности $\Delta_{n+1}$.

Cлучай 2: $Y^{n+1} \backslash Y^{n}=\{k\}$. В этом случае полагаем, что $E_{n+1}$ выполнен в точности на элементах одного класса $\Delta_{k}$-эквивалентных элементов из $\Delta_{k-1}$-класса эквивалентных элементов, содержащего элементы из $E_{n}$, которые лежат в $E_{n} \& \neg B_{n+1}$. Для каждого класса $\Delta_{n}$-эквивалентных элементов, содержащего элементы из $E_{n+1}$, предикат $\Delta_{n+1}$ определяет на пересечении этого класса $\Delta_{n}$-эквивалентных элементов с $E_{n+1}$ точно два класса $\Delta_{n+1}$-эквивалентных элементов, причем предикат для символа $S_{n+1}$ определяем так, что для каждого $x$ из одного из этих $\Delta_{n+1}$-классов эквивалентных элементов $S_{n+1}(x, \bar{z})$ определяют одно и то же подмножество множества $R_{n+1}^{n+2}$, совпадающее с одним из классов $D_{n+1}$-эквивалентности, а для элементов из другого класса - дополнение этого подмножества в $R_{n+1}^{n+2}$. Причем для всех классов, для элементов которых $S_{n+1}(x, \bar{z})$ определено, определимых подмножеств только два и они либо совпадают, либо являются взаимно дополняющими до $R_{n+1}^{n+2}$. Для всех $x$ не из $E_{n+1}$ предикат $S_{n+1}(x, \bar{z})$ ложен при любом $\bar{z}$, а для всех элементов $x$ из $E_{n}$ отношение $S_{k}(x, \bar{z})$ определяет одно и то же подмножество множества $R_{k+1}^{0}(\bar{z})$.

12. Для любых двух различных элементов $x, y$ из $B_{n} \backslash B_{n-1}$, если они $\Delta_{n}$-эквивалентны и $F(x)=F(y)$, то отношение $G_{n}(x, y, u, v)$ задает на множестве $\{a \mid x \leqslant a\} \cup\{b \mid y \leqslant b\}$ отношение эквивалентности так, что оно определяет изоморфизм из $\{a \mid x \leqslant a\}$ на $\{b \mid y \leqslant b\}$ относительно отношений частичного порядка всех эквивалентностей $\Delta_{j}$ и отношений $E_{j}$ для $j \leqslant m$, а также относительно связей с элементами из $C$.

13. Если для элементов $x, y$ из $B_{n} \backslash B_{n-1}$, когда они $\Delta_{n}$-эквивалентны, $F(x)=$ $F(y)$ и отношение $G_{n}(x, y, u, v)$ задает на множестве $\{a \mid x \leqslant a\} \cup\{b \mid y \leqslant b\}$ отношение эквивалентности так, что оно определяет изоморфизм из $\{a \mid x \leqslant a\}$ на $\{b \mid y \leqslant b\}$ относительно отношений частичного порядка всех эквивалентностей и отношений $C_{j}$ для $j \leqslant m$, а элементы $u, v, u \neq v$, переходят при этом изоморфизме в элементы $u^{\prime}, v^{\prime}$ и $F(u)=F(v)$, то $F(u)=F\left(u^{\prime}\right)=F\left(v^{\prime}\right)$, и если $u, v$ лежат в разности $B_{k} \backslash B_{k-1}$ при $k \geqslant n$, то диаграмма из определенных выше $G_{n}\left(x, y, z_{1}, z_{2}\right)$-изоморфизма и изоморфизмов $G_{k}\left(u, v, z_{1}, z_{2}\right)$ и $G_{k}\left(u^{\prime}, v^{\prime}, z_{1}, z_{2}\right)$ будет коммутативна.

14. Определим для элиминации кванторов отношение $\bar{G}_{n}(u, v)$, эквивалентное формуле $(\exists x)(\exists y) \bar{G}_{n}(x, y, u, v)$.

Лемма 5. Теория $T^{m}\left(g_{m}, Y^{m}\right)$ счетно категорична, допускает элиминацию кванторов и разрешима, и множество полных формул у нее вычислимо равномерно по $\mathrm{m}$.

ДокАзАтельство. Доказательство счетной категоричности следует из простой проверки изоморфизма любых двух счетных моделей этой теории челночным методом построения изоморфизма. Нужно заметить, что для конечных подмоделей теории $T^{m}\left(g_{m}, Y^{m}\right)$ выполнено свойство амальгамируемости.

Из счетной категоричности и бесконечности ее моделей следует полнота и разрешимость этой теории. Отсюда и из конечности сигнатуры и элиминации кванторов сразу получаем вычислимость множества ее полных формул. 
Определим теперь теорию $\widehat{T}_{Y}^{* *}=\bigcup \widehat{T}^{m}\left(g_{m}, Y^{m}\right)$.

Лемма 6. Теория $\widehat{T}_{Y}^{* *}$ полна, разрешима и допускает элиминачию кванmоров.

ЛЕмма 7. Любые две счетные модели теории $\widehat{T}_{Y}^{*}$, у которых $A=\bigcup_{n \geqslant 0}\left(\mathscr{D}_{n}\right)_{\mathfrak{M}}$,
зоморфны.

Доказательство следует аналогично доказательству соответствующей леммы из доказательства теоремы 7 (леммы 2) в силу выполнимости аксиом плотности, что гарантирует справедливость свойства амальгамируемости для класса конечных подмоделей моделей с указанными в лемме свойствами. А это позволяет на основе челночного метода [31] построить искомый изоморфизм. ЛЕмма 8. Счетная модель теории $\widehat{T}_{Y}^{*}$, у которой $A=\bigcup_{n \geqslant 0}\left(\mathscr{D}_{n}\right)_{\mathfrak{M}}$, является
простой моделью этой теории.

Доказательство получается непосредственным построением вложения данной модели в любую другую модель этой теории, а из элиминации кванторов сразу следует, что такое вложение будет элементарным вложением.

Из леммы 8 сразу следует неавтоустойчивость данной модели относительно сильных конструктивизаций, так как в случае ее автоустойчивости относительно сильных конструктивизаций по критерию Нуртазина (теорема 1) мы бы смогли распознавать полные формулы, но тогда то же самое можно было бы сделать и в случае фрагмента теории Палютина на фрагменте $C$.

Лемма 9. Счетная модель $\mathfrak{M}$ теории $\widehat{T}_{Y}^{*}$, у которой в множестве $A$ । $\bigcup_{n}\left(\mathscr{D}_{n}\right)_{\mathfrak{M}}$ нет наименьшего элемента, а также существует счетное мно$n \geqslant 0$

жество $Z$ элементов из $B$, удовлетворяющих всем отношениям $E_{n}$ и таких, что для любых $a, b$ выполнено $F(a)=F(b)$, но для любых различных $a, b$ из $Z$ u $n \in \mathbb{N}$ выполнено $\neg(\exists x)(\exists y) G_{n}(x, y, a, b)$, является счетной универсальной моделью этой теории.

Доказательство следует непосредственным построением вложения любой счетной модели этой теории в определенную в лемме модель этой теории, а из элиминации кванторов сразу следует, что такое вложение будет элементарным вложением, и следует ее универсальность.

С помощью леммы 9 мы можем построить подмодель, у которой в множестве $A \backslash \bigcup_{n \geqslant 0}\left(\mathscr{D}_{n}\right)_{\mathfrak{M}}$ есть наименьший элемент $a_{0}$, а также существует элемент $b_{0}$ такой, что $F\left(b_{0}\right)=a_{0}$ и для всех $n$ выполнено $E_{n}\left(b_{0}\right)$, кроме того, для любого элемента $b$ такого, что $F(b)=a_{0}$ и для всех $n$ выполнено $E_{n}(b)$, найдется $k$ такое, что выполнено $G_{k}\left(u, v, b, b_{0}\right)$. В этом случае относительно этого элемента $b_{0}$ уже все предикаты $R_{n}^{0}$ из фрагмента теории Палютина в $C$ делятся на два класса $D_{n}$-эквивалентных элементов отношением $S_{n}\left(b_{0}, \bar{z}\right)$, а в других фрагментах полные формулы определяются с помощью элемента $b_{0}$. Отсюда получаем автоустойчивость этой модели относительно сильных конструктивизаций. 
Неавтоустойчивость относительно сильных конструктивизаций простой модели получаем в силу того, что по критерию Нуртазина (теорема 1) в противном случае множество полных формул было бы вычислимо, а тогда множество $Y$ должно быть рекурсивно, так как во фрагменте $C$ получаем реализацию модели теории Палютина. Если мы рассмотрим теперь простую модель с выделенным элементом $a$, который лежит в $B$, но на котором ложны все $B_{n}$, то модель будет автоустойчивой, так как все отношения во фрагменте $C$ будут делиться на две части и там реализуется теория, эквивалентная базисной, построенной перед доказательством теории Палютина. Счетность числа счетных моделей построенной теории получается за счет того, что отношения из теории Палютина, которые соответствуют элементам из $Y$ за счет введенных предикатов $E_{s}, s \in \mathbb{N}$, попадают в единственный класс эквивалентностей, а элементы из остальных классов могут делить лишь конечное число отношений, связывающих эти элементы из $B$ с наборами во фрагменте теории Палютина на множестве, выделяемом отношением $C$, в отличие от конструкции в доказательстве теоремы Кудайбергенова 7. Аксиомы 12 и 13 гарантируют определенную однородность счетных моделей в конечных обогащениях нужными константами; отсюда и из существования универсальной счетной модели нетрудно получить уже счетность типов изоморфизма счетных моделей. Отсюда следует, что тип изоморфизма счетной модели определяется типом упорядоченного подмножества $A \backslash \bigcup_{n \geqslant 0}\left(\mathscr{D}_{n}\right)_{\mathfrak{M}}$ и количеством независимых элементов типа множества $Z$ из леммы 9.

Теорема 9 доказана.

ТЕОрема 10. Существует разрешимая несчетно категоричная теория такая, что все ее счетные модели сильно конструктивизируемы и неавтоустойчивы относительно сильных конструктивизаций.

ДоказАтельство. Рассмотрим сигнатуру $\Delta$, состоящую из одноместных предикатных символов $\Sigma=\left\{P_{n}^{k} \mid n, k \in \mathbb{N}\right\}$, и рекурсивно перечислимое не рекурсивное множество $A \subseteq \mathbb{N}$.

Пусть $A=\bigcup A_{n}$ и $\left\{A_{n} \mid n \in \mathbb{N}\right\}$ - сильно вычислимая последовательность такая, что

$$
A_{n} \subseteq A_{n+1}, \quad\left|A_{n+1} \backslash A_{n}\right| \leqslant 1
$$

и в $A_{n}$ все элементы меньше $n$.

Пусть $B \subseteq \mathbb{N}$ и все элементы из $B$ меньше $n$, а функция $g: B \rightarrow \mathbb{N}$ разнозначна, причем $g(x)<n$ для любого $x \in B$. Определим теорию $T_{n}^{B, f}$, взяв в качестве сигнатуры $\Sigma_{n}=\left\{P_{\ell}^{k} \mid \ell, k \leqslant n\right\}$.

Выбираем в качестве аксиом следующие.

1. $\neg \exists x\left(P_{\ell}^{0}(x) \& P_{\ell^{\prime}}^{0}(x)\right)$ для $\ell<\ell^{\prime} \leqslant n$.

2. $(\forall x)\left(P_{\ell}^{k+1}(x) \rightarrow P_{\ell}^{0}(x)\right)$ для $\ell \leqslant n, k<n$.

3. $(\exists x)(\exists y)\left(P_{\ell}^{0}(x) \& P_{\ell}^{0}(y) \&(x \neq y) \&(\forall z)\left(P_{\ell}^{0}(z) \Leftrightarrow((z=x) \vee(z=y))\right)\right)$.

4. Если $\ell \notin B$, то $(\forall z)\left(P_{\ell}^{0}(z) \leftrightarrow P_{\ell}^{k+1}(z)\right)$ для всех $k<n$. 
5. Если $\ell \in B$ и $g(\ell)=s$, то $(\forall z)\left(P_{\ell}^{0}(z) \leftrightarrow P_{\ell}^{i}(\bar{z})\right)$ для $i<s$, а для $n \geqslant i \geqslant s$

$$
(\forall z)\left(P_{\ell}^{s}(z) \leftrightarrow P_{\ell}^{i}(z)\right) \&(\exists x)\left(P_{\ell}^{0}(x) \&(\forall z)\left(P_{\ell}^{s}(z) \Leftrightarrow x=z\right)\right) .
$$

Очевидно, что теория $T_{n}^{B, f}$ допускает элиминацию кванторов и счетно категорична.

Определим теперь для любого $A_{n}$ функцию $g_{n}(x), x \in A_{n}$, так, что $g_{n}(x)$ дает наименьший номер $k$ такой, что $x \in A_{k}$.

Определим теорию $T_{A}=\bigcup_{n \geqslant 0} T_{n}^{A_{n}, g_{n}}$.

Сразу же получаем, что теория $T_{A}$ допускает элиминацию кванторов и разрешима. Так как все предикаты истинны не более чем на двух элементах, то $T_{A}$ сильно минимальна и несчетно категорична.

Простая модель будет сильно конструктивизируема, но множество полных формул в любом конечном обогащении константами не вычислимо, так как в противном случае мы смогли бы узнать, полна или нет формула $P_{\ell}^{0}(x)$, а тогда множество $A$ было бы рекурсивным.

Неавтоустойчивость простой модели следует тогда из критерия Нуртазина (теорема 1). Для остальных моделей неавтоустойчивость получаем по следствию о несчетно категоричных теориях.

Теорема 10 доказана.

Алгебраически замкнутые поля характеристики 0 дают пример несчетно категоричной теории, в которой все счетные модели за исключением счетно насыщенной будут автоустойчивы относительно сильных конструктивизаций. Построим еще один пример несчетно категоричных теорий.

Теорема 11. Существует несчетно категоричная теория, у которой простая модель автоустойчива относительно сильных конструктивизаций, а все остальные неавтоустойчивы относительно сильных конструктивизаций.

ДокАЗАТЕЛьСтво. Построим вначале по любому $n$, конечному подмножеству $B \subseteq[0, n[$ и функции $f: B \rightarrow[0, n]$ такой, для любых $x, y \in B$ из $x \neq y$ следует $f(x) \neq f(y)$, счетно категоричную теорию с элиминацией кванторов.

Рассмотрим сигнатуру, состоящую из двухместных предикатов $P_{n}^{k}$ для $n, k \in \mathbb{N}$ и одноместных предикатов $A_{n}, n \in \mathbb{N}$.

Определим теорию кубов, следуя [37].

Определим одноместные предикаты.

0. $(\forall x)\left(A_{k}(x) \rightarrow A_{k+1}(x)\right)$ для $k<n$.

1. $(\forall x)\left(\neg\left(A_{k}(x) \rightarrow(\exists ! y) P_{k}^{0}(x, y) \&(x \neq y)\right)\right)$ и $\left(P_{k}^{0}(x, y) \rightarrow \neg A_{k}(x) \& \neg A_{k}(y)\right)$ для $k \leqslant n$.

2. $(\forall x y)\left(P_{k}^{0}(x, y) \rightarrow P_{k}^{0}(y, x)\right)$ для $k \leqslant n$.

3. $(\forall x y u v)\left(P_{k}^{0}(x, y) \& P_{m}^{0}(x, u) \& P_{m}^{0}(y, v) \rightarrow P_{k}^{0}(u, v)\right)$ для $k, m<n$.

4. $\left(P_{n}^{0}(x, y) \& P_{m}^{0}(x, z)\right) \rightarrow y \neq z$.

5. Если $\ell \notin B$, то для всех $i \leqslant n$ полагаем $(\forall x y)\left(P_{\ell}^{0}(x, y) \leftrightarrow P_{\ell}^{i}(x, y)\right)$. 
Если $\ell \in B$ и $g(\ell)=s$, то полагаем $(\forall x y)\left(P_{\ell}^{0}(x, y) \leftrightarrow P_{\ell}^{i}(x, y)\right)$ для $i<s$, но

$$
\begin{gathered}
(\forall x y)\left(\neg A_{s}(x) \& \neg A_{s}(y)\right) \rightarrow\left(P _ { \ell } ^ { 0 } ( x y ) \leftrightarrow \left(\left(P_{\ell}^{j}(x, y) \vee P_{\ell}^{j}(y, x)\right)\right.\right. \\
\left.\left.\&\left(\neg P_{\ell}^{j}(x, y) \vee \neg P_{\ell}^{j}(y, x)\right)\right)\right), \\
(\forall x y)\left(P_{\ell}^{j}(x, y) \rightarrow \neg P_{\ell}^{j}(y, x)\right), \\
(\forall x y)\left(\left(\neg A_{s}(x) \& \neg A_{s}(y)\right) \rightarrow\left(P_{\ell}^{s}(x, y) \leftrightarrow P_{\ell}^{j}(x, y)\right)\right), \\
(\forall x y u v)\left(\left(\neg A_{s}(x) \& \neg A_{s}(y) \& \neg A_{s}(u) \& \neg A_{s}(v)\right)\right. \\
\left.\rightarrow\left(\left(P_{\ell}^{j}(x, y) \& P_{m}^{0}(x, u) \& P_{m}^{0}(y, v)\right) \rightarrow P_{\ell}^{j}(u, v)\right)\right)
\end{gathered}
$$

для $n>j \geqslant s, m<n$.

6. Множество $A_{k}$ состоит из элементов конечных кубов, в которых нет ребер типа $P_{\ell}^{0}$ для $\ell>k$, а для каждого $0 \leqslant i<n$ в модели имеется единственный куб, у которого для любого $0 \leqslant j \leqslant i$ в модели есть ребра типа $P_{j}^{0}$, а других ребер нет, кроме того, все элементы таких кубов, и только они, лежат в $A_{k}$.

Нетрудно заметить, что теория будет несчетно категорична и разрешима, а простая модель у нее автоустойчива относительно сильных конструктивизаций. Если же мы рассмотрим любую модель $\mathfrak{N}$, не являющуюся простой, но простую над конечным обогащением с вычислимым множеством полных формул, то сможем распознать, когда $P_{\ell}^{0}$ разбивается на две части и не является полной формулой, но тогда $A$ должно быть рекурсивным множеством.

\section{Список литературы}

[1] А. И. Мальцев, "Конструктивные алгебры. I", УМН, 16:3 (1961), 3-60; англ. пер.: A. I. Mal'tsev, "Constructive algebras. I", Russian Math. Surveys, 16:3 (1961), $77-129$.

[2] А.И. Мальцев, "О рекурсивных абелевых группах", Докл. АН CCCP, 146:5 (1962), 1009-1012; англ. пер.: A. I. Mal'tsev, "On recursive Abelian groups", Dokl. Math., 32 (1962), 1431-1434.

[3] A. Fröhlich, J. C. Shepherdson, "Effective procedures in field theory", Philos. Trans. Roy. Soc. London. Ser. A, 248:950 (1956), 407-432.

[4] Ю.Л. Ершов, "Конструктивные модели", Избранные вопросы алгебры и логики, Наука, Новосибирск, 1973, 111-130.

[5] M. Morley, "Decidable models", Israel J. Math., 25:3-4 (1976), 233-240.

[6] А.Т. Нуртазин, "Сильные и слабые конструктивизации и вычислимые семейства", Алгебра и логика, 13:3 (1974), 311-323; англ. пер.: А. T. Nurtazin, "Strong and weak constructivizations, and enumerable families", Algebra Logic, 13:3 (1974), 177-184.

[7] С. С. Гончаров, Ю. Л. Ершов, Конструктивные модели, Научная книга, Новосибирск, 1999, ISBN: 5-88119-021-1, 360 с.; англ. пер.: Yu. L. Ershov, S. S. Goncharov, Constructive models, Siberian School of Algebra and Logic, Consultants Bureau, New York, 2000, ISBN: 0-306-11066-0, xii+293 pp.

[8] C. J. Ash, T.S. Millar, "Persistently finite, persistently arithmetic theories", Proc. Amer. Math. Soc., 89:3 (1983), 487-492. 
[9] Е.Б. Фокина, "О спектрах вычислимых моделей", Вестн. НГУ. Сер. матем., мех., информ., 6:4 (2006), 69-73.

[10] E. B. Fokina, "Arithmetic Turing degrees and categorical theories of computable models", Mathematical logic in Asia, World Sci. Publ., Hackensack, NJ, 2006, 58-69.

[11] A. Gavryushkin, "On complexity of Ehrenfeucht theories with computable model", Logical Approaches to Computational Barriers (Second Conference on Computability in Europe), Report Series, Swansea Univ., 2006, 105-108.

[12] А.Н. Гаврюшкин, "Сложность эренфойхтовых моделей", Алгебра и логика, 45:5 (2006), 507-519; англ. пер.: A. N. Gavryushkin, "Complexity of Ehrenfeucht models", Algebra Logic, 45:5 (2006), 289-295.

[13] A. Gavryushkin, "Computable models spectra of Ehrenfeucht theories", Logic Colloquium 2007, Book of Abstracts, Uniwersytet Wrocławski, 2007, 46-47.

[14] А.Н. Гаврюшкин, "Спектры вычислимых моделей эренфойхтовых теорий”, Алгебра и логика, 46:3 (2007), 275-289; англ. пер.: А. N. Gavryushkin, "Spectra of computable models for Ehrenfeucht theories", Algebra Logic, 46:3 (2007), 149-157.

[15] A. Gavryushkin, "Computable models spectra of Ehrenfeucht theories", Logic and Theory of Algorithms (Fourth Conference on Computability in Europe), CiE 2008 Local Proceedings, University of Athens, 2008, 50-51.

[16] А.Н. Гаврюшкин, "О конструктивных моделях теорий с линейным порядком Рудина-Кейслера", Вестн. НГУ. Сер. матем., мех., информ., 9:2 (2009), 30-37.

[17] S. Goncharov, B. Khoussainov, "Open problems in the theory of constructive algebraic systems", Computability theory and its applications (Boulder, CO, 1999), Contemp. Math., 257, Amer. Math. Soc., Providence, RI, 2000, 145-170.

[18] J. F. Knight, "Nonarithmetical $\aleph_{0}$-categorical theories with recursive models", J. Symbolic Logic, 59:1 (1994), 106-112.

[19] S. Lempp, T. Slaman, "The complexity of the index sets of $\aleph_{0}$-categorical theories and of Ehrenfeucht theories", Advances in logic, Contemp. Math., 425, Amer. Math. Soc., Providence, RI, 2007, 43-47.

[20] T. S. Millar, "Foundations of recursive model theory", Ann. Math. Logic, 13:1 (1978), 45-72.

[21] T. Millar, "Persistently finite theories with hyperarithmetic models", Trans. Amer. Math. Soc., 278:1 (1983), 91-99.

[22] T.S. Millar, "Decidability and the number of countable models", Ann. Pure Appl. Logic, 27:2 (1984), 137-153.

[23] T. Millar, "Decidable Ehrenfeucht theories", Recursion theory (Ithaca, NY, 1982), Proc. Sympos. Pure Math., 42, Amer. Math. Soc., Providence, RI, 1985, 311-321.

[24] E. A. Palyutin, "On algebras of formulas for denumerably categorical theories", Colloq. Math., 31:2 (1974), 157-159.

[25] М. Г. Перетятькин, "О полных теориях с конечным числом счетных моделей”, $A л$ гебра и логика, 12:5 (1973), 550-576; англ. пер.: М. G. Peretyat'kin, "On complete theories with a finite number of denumerable models", Algebra Logic, 12 (1973), 310-326.

[26] R. C. Reed, "A decidable Ehrenfeucht theory with exactly two hyperarithmetic models", Ann. Pure Appl. Logic, 53:2 (1991), 135-168.

[27] J.H. Schmerl, "A decidable $\aleph_{0}$-categorical theory with a nonrecursive Ryll-Nardzewski function", Fund. Math., 98:2 (1978), 121-125.

[28] R. L. Vaught, "Denumerable models of complete theories", Infinitistic Methods (Proc. Sympos. Foundations of Math., Warsaw, 1959), Pergamon, Oxford; Państwowe Wydawnictwo Naukowe, Warszawa, 1961, 303-321.

[29] С. С. Гончаров, Счетные булевы алгебры и разрешимость, Сибирская школа алгебры и логики, Научная книга, Новосибирск, 1996, ISBN: 5-88119-004-1, 362 с.; 
англ. пер.: S.S. Goncharov, Countable Boolean algebras and decidability, Siberian School Algebra Logic, Consultants Bureau, New York, 1997, ISBN: 0-306-11061-X, $318 \mathrm{pp}$.

[30] Х. Роджерс, Теория рекурсивных функиий и эффективная вычислимость, Мир, M., 1972, 624 с.; пер. с англ.: H. Rogers Jr., Theory of recursive functions and effective computability, McGraw-Hill, Toronto-London-New York, 1967, 482 pp.

[31] Г. Кейслер, Ч. Ч. Чэн, Теория моделей, Мир, М., 1977, 614 с.; пер. с англ.: C. C. Chang, H. J. Keisler, Model theory, Stud. Logic Found. Math., 73, North-Holland, Amsterdam-London; American Elsevier, New York, 1973, 550 pp.

[32] А. И. Мальцев, Алгоритмы и рекурсивные функиии, Наука, М., 1965, 391 с.; англ. пер.: A. I. Mal'tsev, Algorithms and recursive functions, Wolters-Noordoff, Groningen, 1970, $372 \mathrm{pp}$.

[33] С. С. Гончаров, А. Т. Нуртазин, "Конструктивные модели полных разрешимых теорий”, Алгебра и логика, 12:2 (1973), 125-142; англ. пер.: S. S. Goncharov, A. T. Nurtazin, "Constructive models of complete solvable theories", Algebra Logic, 12 (1974), 67-77.

[34] L. Harrington, "Recursively presented prime models", J. Symbolic Logic, 39:2 (1974), 305-309.

[35] С. С. Гончаров, "Автоустойчивость простых моделей относительно сильных конструктивизаций”, Алгебра и логика, 48:6 (2009), 729-740; англ. пер.: S. S. Goncharov, "Autostability of prime models under strong constructivizations", Algebra Logic, 48:6 (2009), 410-417.

[36] К.Ж. Кудайбергенов, “Автоустойчивость и продолжение конструктивизаций”, Сиб. матем. журн., 25:5 (1984), 72-78; англ. пер.: K. Zh. Kudaibergenov, "Autostability and extension of constructivizations", Siberian Math. J., 25:5 (1984), 743-749.

[37] B. Khoussainov, A. Nies, R. Shore, "Computable models of theories with few models", Notre Dame J. Formal Logic, 38:2 (1997), 165-178.

C. C. Гончаров (S.S. Goncharov)

Поступила в редакцию

Институт математики

29.06 .2010

им. С. Л. Соболева СО РАН

E-mail: gonchar@math.nsc.ru 